\title{
Elevating expression of MeCP2 T158M rescues DNA binding and Rett syndrome-like phenotypes
}

\author{
Janine M. Lamonica, ${ }^{1}$ Deborah Y. Kwon, ${ }^{1}$ Darren Goffin, ${ }^{1}$ Polina Fenik, ${ }^{2}$ Brian S. Johnson, ${ }^{1}$ Yue Cui,, ${ }^{1}$ Hengyi Guo, ${ }^{1}$ \\ Sigrid Veasey, ${ }^{2}$ and Zhaolan Zhou ${ }^{1}$ \\ 'Department of Cenetics, ${ }^{2}$ Center for Sleep and Circadian Neurobiology, Department of Medicine, Perelman School of Medicine, University of Pennsylvania, Philadelphia, Pennsylvania, USA
}

\begin{abstract}
Mutations in the X-linked gene encoding methyl-CpG-binding protein 2 (MeCP2) cause Rett syndrome (RTT), a neurological disorder affecting cognitive development, respiration, and motor function. Genetic restoration of MeCP2 expression reverses RTT-like phenotypes in mice, highlighting the need to search for therapeutic approaches. Here, we have developed knockin mice recapitulating the most common RTT-associated missense mutation, MeCP2 T158M. We found that the T158M mutation impaired MECP2 binding to methylated DNA and destabilized MeCP2 protein in an age-dependent manner, leading to the development of RTT-like phenotypes in these mice. Cenetic elevation of MeCP2 T158M expression ameliorated multiple RTTlike features, including motor dysfunction and breathing irregularities, in both male and female mice. These improvements were accompanied by increased binding of MeCP2 T158M to DNA. Further, we found that the ubiquitin/proteasome pathway was responsible for MeCP2 T158M degradation and that proteasome inhibition increased MeCP2 T158M levels. Together, these findings demonstrate that increasing MeCP2 T158M protein expression is sufficient to mitigate RTT-like phenotypes and support the targeting of MeCP2 T158M expression or stability as an alternative therapeutic approach.
\end{abstract}

\section{Introduction}

Rett syndrome (RTT) is one of the most common forms of intellectual disability in young girls. After a period of normal development, the disease emerges by the first 6-18 months of age and is characterized by loss of learned language skills, deceleration of head growth, irregular breathing patterns, stereotypical hand-wringing, and profound cognitive impairment (1). RTT is a disorder of known genetic etiology, with the vast majority of cases the result of de novo mutations in the methyl-CpG-binding protein 2 (MECP2) gene (2). MeCP2 binds preferentially to symmetrically methylated $\mathrm{CpG}$ dinucleotides via its methyl-CpG-binding domain (MBD) and is thought to mediate transcriptional repression through the recruitment of corepressors, such as the SIN3 transcription regulator family member A (SIN3A) and the nuclear receptor corepressor (NCoR) and silencing mediator of retinoic acid and thyroid hormone receptor (SMRT) corepressor complexes (3-7). Recent studies, however, have revealed that MeCP2-dependent gene regulation is much more complex, with MeCP2 activating or repressing gene expression depending on the cellular context, gene length, and signatures of DNA methylation (8-14).

Genetic reexpression of MeCP2 in symptomatic Mecp2-null mice leads to restoration of neuronal function and reversal of phenotypes, demonstrating that RTT is treatable, at least in mice, even at late stages of the disease (15). One leading approach to clinically restore $\mathrm{MeCP} 2$ function in patients is via gene therapy (16), a challenging endeavor, because MeCP2 expression levels

Conflict of interest: The authors have declared that no conflict of interest exists.

Submitted: October 17, 2016; Accepted: February 9, 2017.

Reference information: J Clin Invest. 2017;127(5):1889-1904.

https://doi.org/10.1172/JCI90967. must be precisely controlled, as both over- and underexpression lead to neurological phenotypes in humans and mice (17-22). In addition, gene therapy approaches may have limited feasibility in the case of dominant-negative mutations. Thus, a genotype-specific approach is needed to model the disorder and determine how different RTT-causing mutations disrupt MeCP2 function.

The majority of missense mutations identified in patients with RTT are clustered in the MBD and transcription repression domain (TRD) of MeCP2, with one of the most frequent mutations occurring at residue $\mathrm{T} 158$, converting it to a methionine (MeCP2 T158M) or, in rare cases, alanine (T158A) (23). To determine the genetic contribution of the MeCP2 T158M mutation to RTT, we developed MeCP2 T158M-knockin (Mecp2 ${ }^{\text {T158M }}$ ) mice and found that they recapitulate many clinical features of RTT. We further found that MeCP2 T158M levels failed to increase during postnatal development, contributing, at least in part, to its reduced DNA binding. This raised the possibility that stabilizing MeCP2 T158M protein or increasing its expression might ameliorate disease phenotypes. To test this in vivo, we generated a Tg mouse line expressing MeCP2 T158M throughout the CNS and found that elevation of MeCP2 T158M expression restored the binding of mutant MeCP2 to DNA and, notably, improved many features of the disease. Furthermore, overexpression of MeCP2 T158M in Tg mice did not lead to the development of RTT-like phenotypes, ruling out the possibility that MeCP2 T158M shows dominant-negative effects. Finally, we found that the levels of MeCP2 T158M can be increased pharmacologically with proteasome inhibitors. Together, these findings demonstrate that reduced levels of MeCP2 T158M at least partially underlie RTT pathology and provide a proof of principle that pharmacologic elevation or stabilization of MeCP2 protein represents an effective approach to treating patients with MBD missense mutations. 

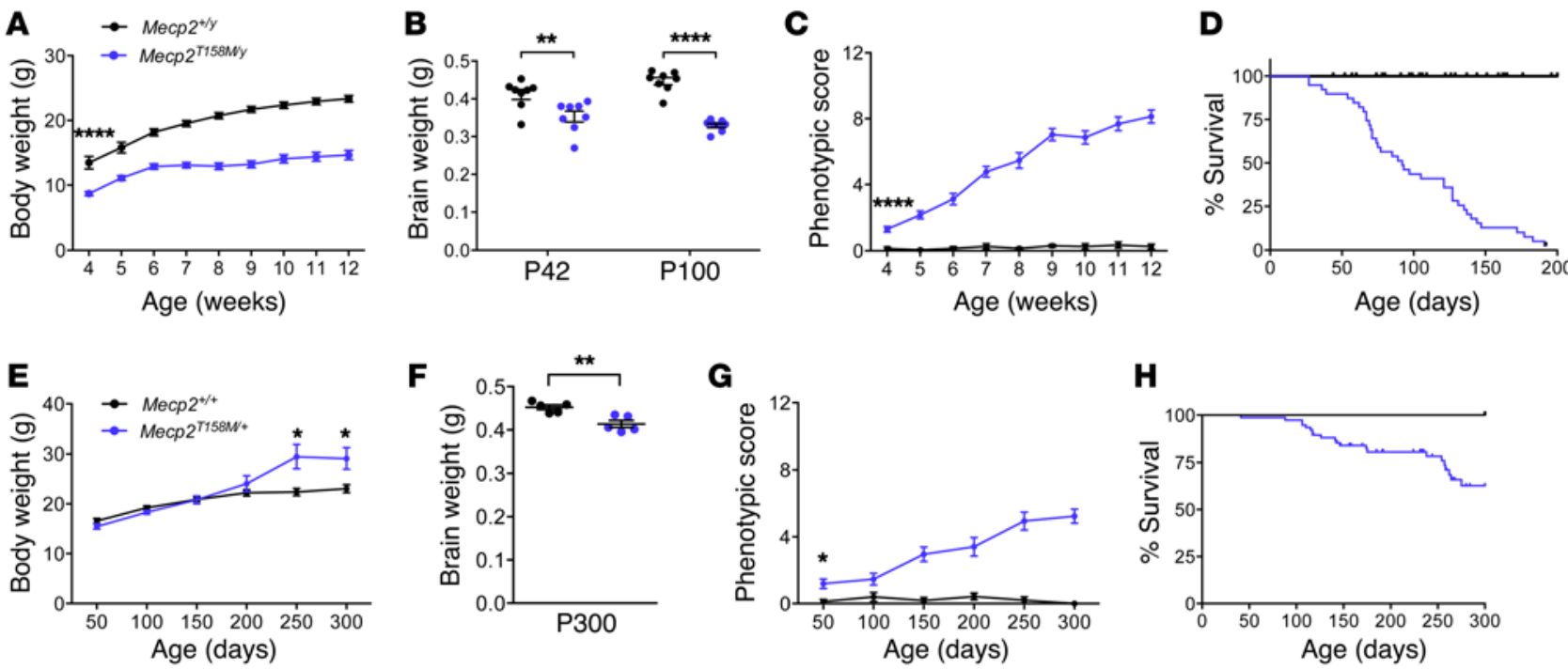

Figure 1. Mecp2 $2^{T 158 M}$ mice display RTT-like phenotypes. (A) Body weights of Mecp2 ${ }^{T 158 M / y}$ male mice $\left(n=53\right.$, blue) were reduced relative to Mecp2 $2^{+/ y}$ littermate body weights ( $n=29$, black) at 4 weeks and thereafter. ${ }^{* * *} P<0.0001$, by Student's $t$ test. (B) Mecp $2^{T 158 M / y}$ male mice had reduced brain weights at P42 and P100 ( $n=8$ for both genotypes and ages). ${ }^{* *} P<0.01$ and ${ }^{* * *} P<0.0001$, by Student's $t$ test with Bonferroni's post-hoc test. (C) Age-dependent presentation of RTT-like phenotypes in Mecp $2^{T 158 M / y}(n=45)$ versus Mecp2 ${ }^{+/ y}(n=24)$ male mice. ${ }^{* * *} P<0.0001$, by Student's $t$ test. (D) Kaplan-Meier survival curve revealed that Mecp2 $2^{T 158 M / y}$ male mice had premature lethality (Mecp2 $2^{+/ y}$ median survival $>200$ days, $n=35$, vs. Mecp2 $2^{T 158 M / y}$ median survival $=92$ days, $n=39)$. (E) Body weight versus postnatal age for female Mecp $2^{T 158 M /+}$ mice $(n=12)$ compared with Mecp2 $2^{+/+}$littermates $(n=10) . M e c p 2^{T 158 M /+}$ mice had significantly higher body weights at P250 or older. ${ }^{*} P<0.05$, by Student's $t$ test. (F) Brain weights of female mice at P300 ( $n=5$ per genotype). ${ }^{* *} P<0.01$, by Student's $t$ test with Bonferroni's post-hoc test. (G) Phenotypic scores of female Mecp2 $2^{T 158 M /+}$ mice $(n=12)$ relative to scores for Mecp2 $2^{+/+}$littermates $(n=10) .{ }^{*} P<0.05$, by Student's $t$ test. (H) Reduced survival of $\operatorname{Mecp}^{T 158 M /+}(n=76)$ compared with Mecp2 ${ }^{+/+}(n=14)$ female mice. Censored animals are indicated by a tick mark. All error bars represent the mean \pm SEM.

\section{Results}

Development and phenotypic characterization of $M e c p 2^{T 158 M}$ mice. $\mathrm{MeCP} 2 \mathrm{~T} 158 \mathrm{M}$ is the most common missense mutation identified in individuals with classical RTT (23). To examine the role of this mutation in RTT etiology, we used a homologous recombination approach and generated knockin mice recapitulating this mutation (Mecp2 $2^{T 158 M}$ mice) (Supplemental Figure 1A; supplemental material available online with this article; https://doi.org/10.1172/ JCI90967DS1). To facilitate the identification of mutant MeCP2 protein, we fused a Tavi affinity tag (TEV protease cleavage and avidin binding) onto the C-terminus of MeCP2, allowing it to be biotinylated in vivo and identified with streptavidin (Supplemental Figure 1A). WT MeCP2-Tavi mice are phenotypically indistinguishable from WT mice up to 20 weeks of age, and both the tagged and untagged WT protein bind similarly to DNA, demonstrating that the tag itself does not affect MeCP2 function (B.S. Johnson, unpublished observations). The presence of the T158M mutation was confirmed by sequencing (Supplemental Figure 1B) and probing brain lysates with a T158 site-specific antibody (Supplemental Figure 1C).

We next monitored T158M-knockin mice for the development of RTT-like phenotypes. Consistent with previous findings in Mecp2-null mice $(24,25), M e c p 2^{T 158 M / y}$ male mice weighed significantly less (Figure $1 \mathrm{~A}$ ) and had significantly reduced brain weights compared with Mecp $2^{+/ y}$ male littermates (Figure 1B). Phenotypic scoring, which measures a range of RTT-like phenotypes, including gait abnormalities, motor deficits, and breathing (15), was performed on Mecp $2^{T 158 M / y}$ mice aged 4-12 weeks. We observed a marked, age-dependent increase in the phenotypic score for $M e c p 2^{T 158 M / y}$ male mice leading to premature death at approximately 13 weeks (Figure 1, C and D).
Since heterozygous females are the more clinically relevant mouse model, we also characterized RTT-like phenotypes in female mice. We found that $M e c p 2^{T 158 M /+}$ heterozygous females weighed significantly more than did Mecp $2^{+/+}$female littermates after 8 months of age (Figure 1E). Despite their increased body weight, $M e c p 2^{T 158 M /+}$ female mice were microcephalic (Figure 1F). Compared with hemizygous Mecp2 $2^{T 158 M / y}$ male mice, heterozygous Mecp2 $2^{T 158 M /+}$ female mice developed similar RTT-like phenotypes, though they were less severe and had delayed progression (Figure 1G). In addition, $M e c p 2^{T 158 M /+}$ females lived considerably longer than did $M e c p 2^{T 158 M / y}$ males, but also died prematurely, occasionally as a result of tonicclonic seizures after routine handling (Figure 1H). Thus, both male and female $M e c p 2^{T 158 M}$ mice display phenotypes similar to those of Mecp2-null mice $(24,25)$ and recapitulate many of the neurological deficits reminiscent of RTT.

MeCP2 T158M binds to DNA in a protein level-dependent manner. T158 is located in the C-terminal portion of the MBD and is critical for stabilizing the tandem Asx-ST motifs and increasing the affinity of MeCP2 for methylated DNA (26). Further, it has been reported that mutation at $\mathrm{T} 158$ reduces the affinity of MeCP2 for methylated DNA, leading to the pathogenesis of RTT $(27,28)$. Consistent with this notion, we found that MeCP2 T158M protein was diffusely localized in the nucleus, suggesting impaired binding of MeCP2 T158M to DNA (Figure 2A). However, in addition, we observed that the protein expression level of MeCP2 T158M was approximately $82 \%$ of WT MeCP2 expression at PO or P1 (Supplemental Figure 1B and Supplemental Figure 2B), but decreased to approximately $50 \%$ of WT levels at P7 and further decreased to approximately $35 \%$ of WT levels at P14, where it remained 
A

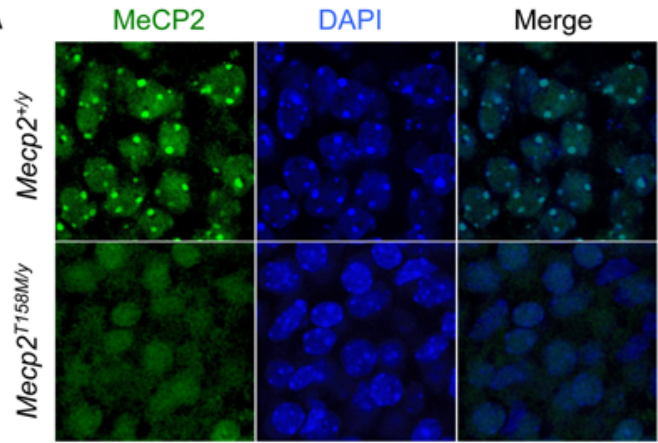

B

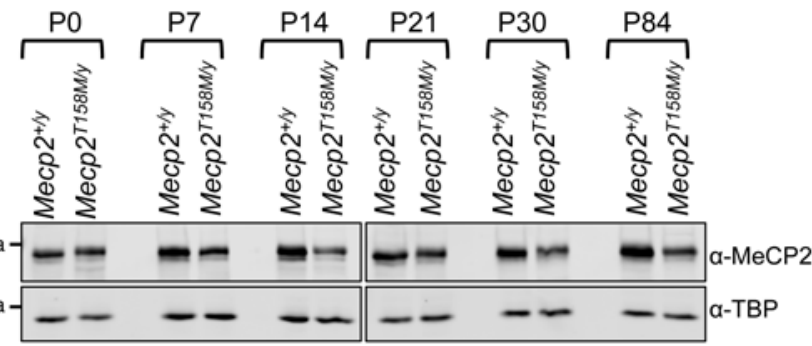

- $M e c p 2^{+/ y}$ - Mecp2 $2^{T 158 M / y}$
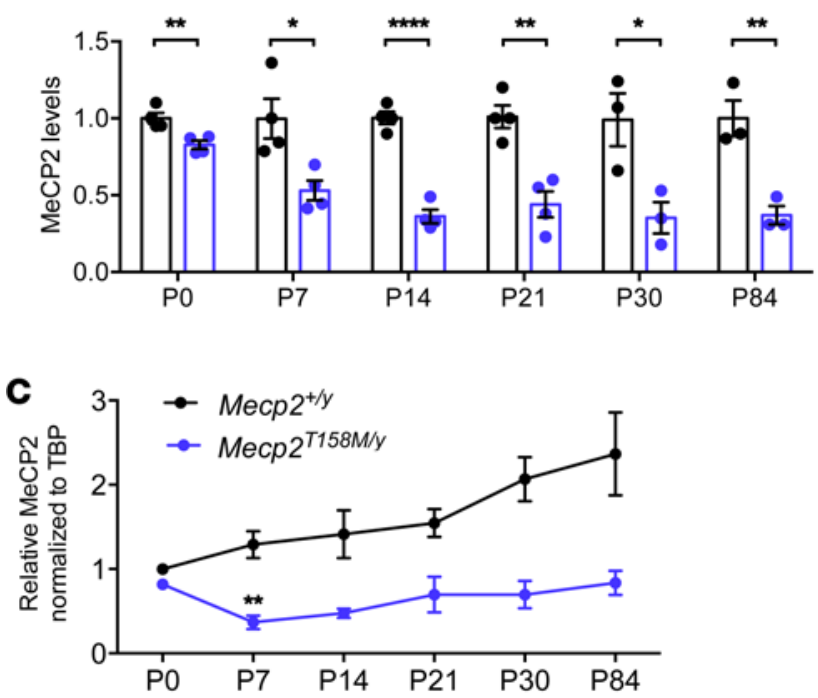

Figure 2. The binding of MeCP2 T158M to DNA is protein expression level dependent. (A) Immunohistochemical staining for MeCP2 in the cortex of male Mecp2 ${ }^{T 158 M / y}$ mice compared with Mecp2 $2^{+/ y}$ littermates at P30. MeCP2 T158M staining is largely diffuse, without visible foci. Original magnification: $\times 100$. (B) MeCP2 protein levels in whole brain from Mecp2 $2^{1158 M / y}$ mice at P0, P7, P14, P21, $\mathrm{P} 30$, and P84, as compared with levels in Mecp2 ${ }^{+/ y}$ controls ( $n=3-4$ per genotype). Quantification is relative to WT MeCP2 levels at each age and normalized to TBP to account for differences in loading. (C) MeCP2 protein levels in whole brain from Mecp2 $2^{T 158 M / y}$ and $M e c p 2^{+/ y}$ animals during development. Quantification is relative to $\mathrm{PO}$ for each genotype and normalized to TBP ( $n=3$ per genotype and age). Despite similar protein levels at PO, WT MeCP2 levels steadily increased, while MeCP2 T158M levels declined and never rose to the levels of WT MeCP2 in adulthood. (D) ChIP-qPCR for MeCP2 and control IgG in whole brain from Mecp2 $2^{+/ y}$ and Mecp2 2158M/y littermates at PO (top, $n=5$ per genotype), P14 (middle, $n=3$ per genotype), and P30 (bottom, $n=4$ per genotypes). Binding of MeCP2 T158M to DNA was similar to that of WT MeCP2 at P0 but was reduced at P14 and P30. (E) ChIP-qPCR as in $\mathbf{D}$ plotted across age. Binding of MeCP2 T158M to DNA failed to increase with postnatal age. Statistical analyses in $\mathbf{B}-\mathbf{D}$ were done by unpaired Student's $t$ test. Comparisons in $\mathbf{E}$ were done by 1-way ANOVA, followed by Tukey's post-hoc test. ${ }^{*} P<0.05,{ }^{* *} P<0.01,{ }^{* * *} P<0.001$, and ${ }^{* * *} P<0.0001$. All error bars represent the mean \pm SEM.
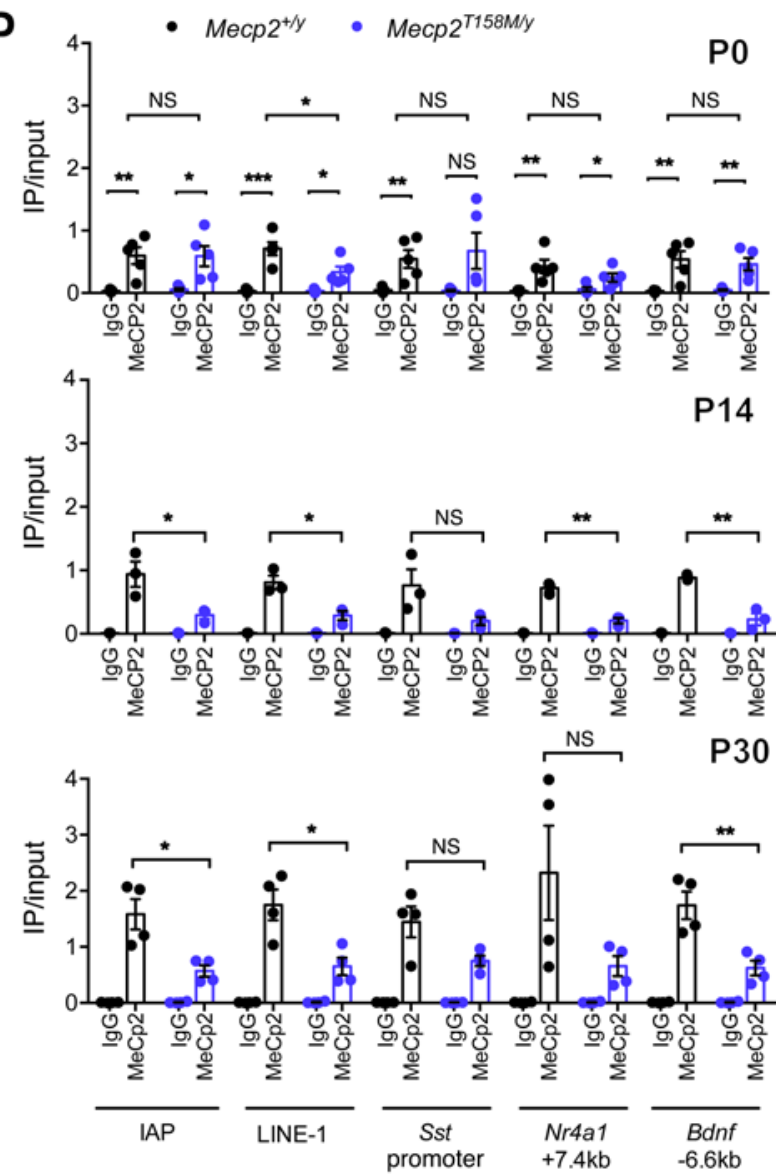

E

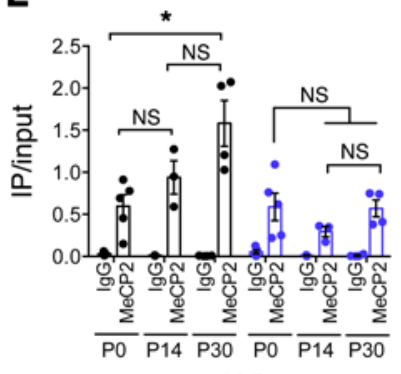

IAP

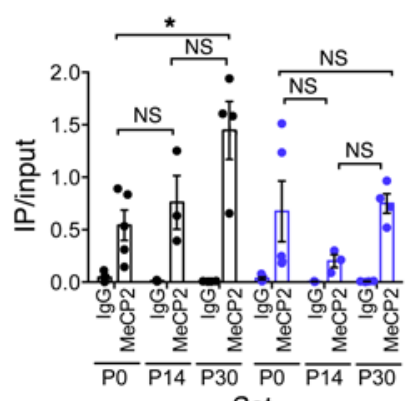

Sst

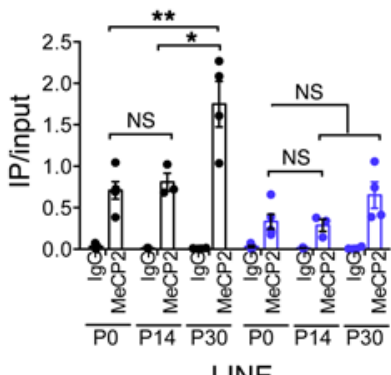

LINE

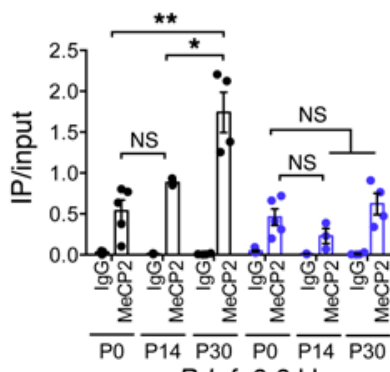

Bdnf $-6.6 \mathrm{~kb}$ 
relatively constant until 12 weeks of age (Figure 2B). In contrast, Mecp2 T158M mRNA expression remained unchanged over this age range (Supplemental Figure 2A), suggesting increased protein turnover. MeCP2 protein levels were also found to be reduced in fibroblasts derived from a female patient with RTT harboring the T158M mutation (27), in the absence of any change in mRNA (Supplemental Figure 2B). To determine whether MeCP2 T158M has reduced protein stability, we cultured cortical neurons isolated from Mecp $2^{T 158 M / y}$ and Mecp $2^{+/ y}$ PO pups and treated the neurons with cycloheximide ( $\mathrm{CHX}$ ) to block new protein synthesis. Whereas WT MeCP2 protein expression remained unchanged upon $\mathrm{CHX}$ treatment, MeCP2 T158M protein was reduced by approximately 75\% after a 10-hour treatment, suggesting decreased MeCP2 T158M protein stability (Supplemental Figure 2C). Moreover, we found that WT MeCP2 protein levels steadily increased postnatally, whereas MeCP2 T158M levels failed to increase at P7 and never reached those of WT MeCP2 in adulthood, despite similar protein expression levels in newborn pups (Figure 2C).

We hypothesized that the reduced levels of MeCP2 T158M protein might itself contribute to the impaired DNA binding and the consequent progression of disease phenotypes seen in Mecp $2^{T 158 M}$ animals. To investigate how MeCP2 T158M protein levels impact DNA binding, we performed ChIP, followed by quantitative PCR (ChIP-qPCR) throughout early development, comparing WT MeCP2 and MeCP2 T158M binding at a number of genomic loci that were found to be bound by $\mathrm{MeCP} 2$ in previous studies, including the promoters of intercisternal A particles (IAPs), L1 retrotransposons (LINE-1 RNAs), somatostatin (Sst), and the upstream and downstream regions of brain-derived neurotrophic factor (Bdnf) and nerve growth factor ( $N r 4 a 1)$, respectively $(29,30)$. At PO, an age at which the levels of WT MeCP2 and MeCP2 T158M are most similar (Figure 2B), MeCP2 binding at each target loci was comparable between $M e c p 2^{+/ y}$ and $M e c p 2^{\text {T158M/y }}$ animals (Figure 2D), suggesting that MeCP2 T158M retains partial DNA-binding affinity, in agreement with previous in vitro studies (31). At P14, WT MeCP2 showed an overall increase in binding relative to that detected at PO, and binding was further increased at P30 (Figure 2, D and E), consistent with the steady rise in WT MeCP2 levels during development (Figure 2C). By contrast, the binding of MeCP2 T158M to DNA was significantly reduced relative to WT MeCP2 binding at both P14 and P30 at several loci (Figure 2, D and E), coincident with the overall reduced levels of this mutant protein at these ages (Figure 2B). These data suggest that the binding of MeCP2 T158M to DNA is at least in part dependent on its protein level. We further tested this in cultured cells by engineering Neuro2a (N2a) clonal cell lines stably expressing either WT MeCP2 or MeCP2 T158M. We also tagged both WT and mutant MeCP2 with an N-terminal FLAG tag and a C-terminal fusion of Dendra2, a photoconvertible protein similar to GFP (FLAG-MeCP2-Dendra2 and FLAGT158M-Dendra2, respectively) to facilitate the visualization of MeCP2 (Supplemental Figure 2D). We then generated clonal lines that expressed varying levels of FLAG-MeCP2-Dendra2 or FLAGT158M-Dendra2 protein (Supplemental Figure 2E). Using immunofluorescence to examine MeCP2 localization, we found that WT MeCP2 colocalized with heterochromatic foci in both lowand high-expressing FLAG-MeCP2-Dendra2 cells, while FLAGT158M-Dendra2, when expressed at low levels, remained largely diffuse (Supplemental Figure 2F). Interestingly, in high-expressing FLAG-T158M-Dendra2 cell lines, MeCP2 localized to heterochromatic foci with increased frequency. Together, these results demonstrate that MeCP2 T158M is capable of binding to methylated DNA in a protein level-dependent manner. Mutation at T158 partially reduced the binding affinity of $\mathrm{MeCP} 2$ for methylated DNA in vivo and in vitro.

Generation and characterization of $\mathrm{T} 158 \mathrm{M}-\mathrm{Tg}$ mice. Since high-level expression of MeCP2 T158M in cultured cells rescues DNA binding, we examined the possibility that elevation of mutant MeCP2 expression in mice might restore MeCP2 function and ameliorate disease phenotypes. To test this, we first generated $\mathrm{Tg}$ mice that express MeCP2 T158M under the control of the mouse prion promoter (Prp), which drives broad expression in the CNS (32) (Figure 3A). Given that the e1 isoform of MeCP2 is abundantly expressed in the brain $(33,34)$ and that knockout of this isoform fully recapitulates RTT-like phenotypes in mice (35), we generated Tg mice expressing MeCP2-e1 with the T158M mutation (T158M$\mathrm{Tg}$ ). In addition, we included an N-terminal FLAG tag, allowing us to distinguish between endogenous and $\mathrm{Tg} \mathrm{MeCP} 2$ protein. We obtained $2 \mathrm{Tg}$ founder lines expressing Tg MeCP2 T158M protein at levels comparable to endogenous WT MeCP2 levels (Figure 3B). The presence of the $\mathrm{T} 158 \mathrm{M}$ mutation was confirmed using a T158 site-specific antibody (Figure $3 \mathrm{C}$ ). We found that $\mathrm{Tg} \mathrm{T} 158 \mathrm{M}$ protein was highly expressed in the brain, with little to no detectable expression in the lung, liver, spleen, heart, kidney, or small intestine (Supplemental Figure 3A). Within the brain, Tg T158M protein exhibited widespread distribution in different brain regions that was comparable to the expression pattern of endogenous MeCP2 and consistent with the broad activity of the prion promoter throughout the CNS (Figure 3D, and Supplemental Figure 3, B and C). Moreover, the expression of $\mathrm{Tg} \mathrm{MeCP} 2 \mathrm{~T} 158 \mathrm{M}$ protein remained relatively stable throughout development (Figures $3 \mathrm{E}$ and Supplemental Figure $3 \mathrm{D})$ and retained the ability to interact with known binding partners, such as SIN3A, transducin $\beta$-like related protein (TBLR1), and histone deacetylase 3 (HDAC3) (Figure 3F).

T158M-Tg mice that overexpress WT MeCP2 by approximately 1 -fold have enhanced motor and contextual learning by 10 weeks of age, with seizures and hypoactivity becoming apparent by 20 weeks of age and premature death occurring in 30\% of animals by 1 year of age (19). To examine whether T158M-Tg mice manifest a similar neurological phenotype, we monitored male $\mathrm{T} 158 \mathrm{M}-\mathrm{Tg}$ mice $\left(\mathrm{Mecp}^{+/ y} \mathrm{~T} 158 \mathrm{M}-\mathrm{Tg}\right)$ over a period of 29 weeks (200 days). We found that Mecp2 $2^{+/ y} \mathrm{~T} 158 \mathrm{M}-\mathrm{Tg}$ male mice were indistinguishable from their WT littermates with respect to body weight, brain weight, phenotypic score, and lifespan (Figure 3, G-J). Notably, Mecp $2^{+/ y}$ T158M-Tg mice were viable until 2 years of age, with no premature death (data not shown). Female T158M-Tg mice (Mecp2 $2^{+/+} \mathrm{T} 158 \mathrm{M}-\mathrm{Tg}$ ) were also comparable to WT $\mathrm{Mecp2}^{+/+}$ females in terms of body weight and phenotypic score (Supplemental Figure 3, E and F). Thus, our findings in both knockin and Tg mice suggest that MeCP2 T158M is a loss-of-function mutation, at least partially, and does not show dominant-negative effects.

Increasing MeCP2 T158M expression ameliorates RTT-like phenotypes in Mecp $2^{T 158 M / y}$ male mice. The generation of $\mathrm{Tg}$ mice expressing MeCP2 T158M without dominant-negative effects allowed us to test the possibility that increased expression of the 
A

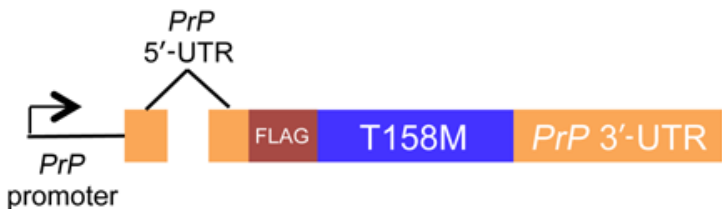

B

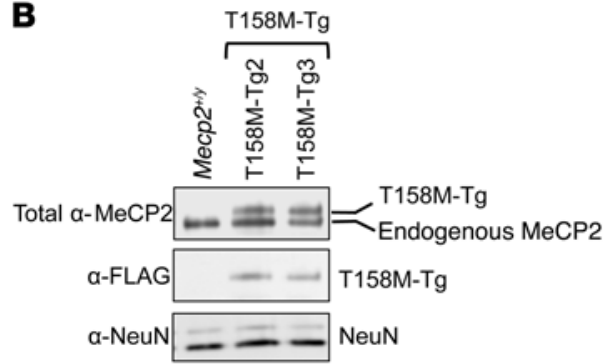

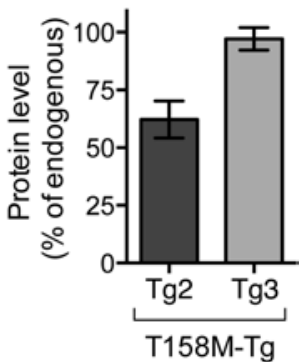

C

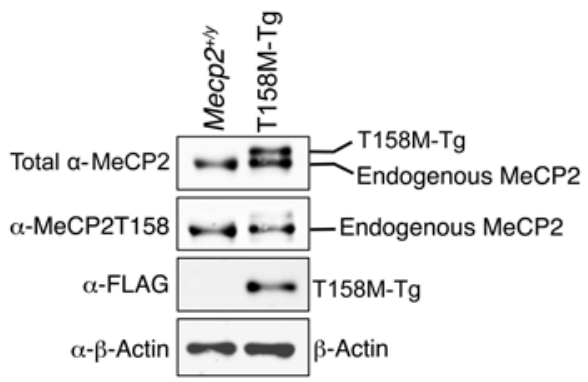

D

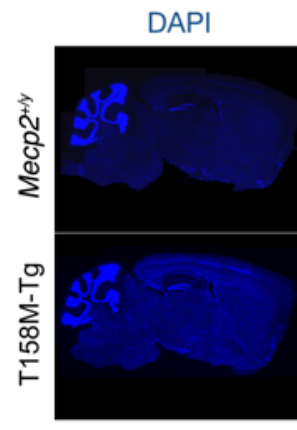

MeCP2

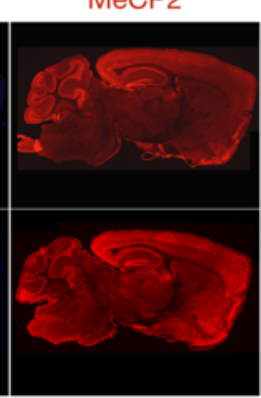

FLAG

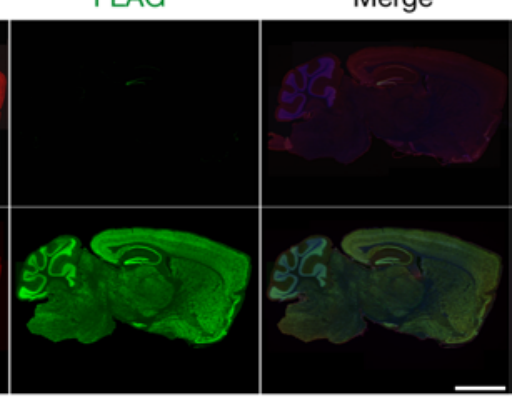

E
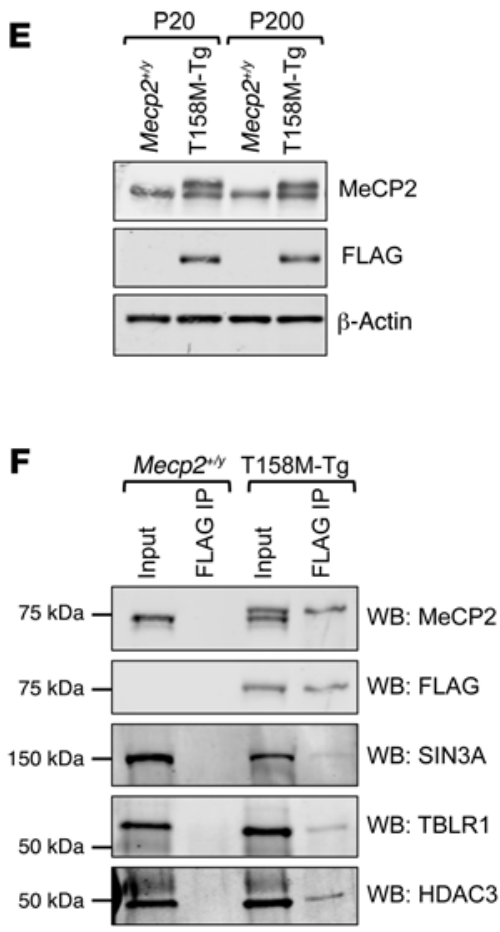

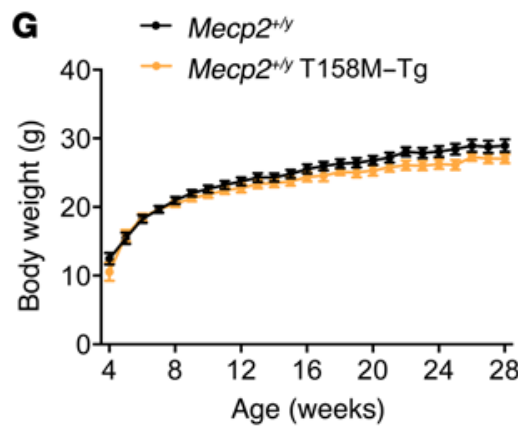

1

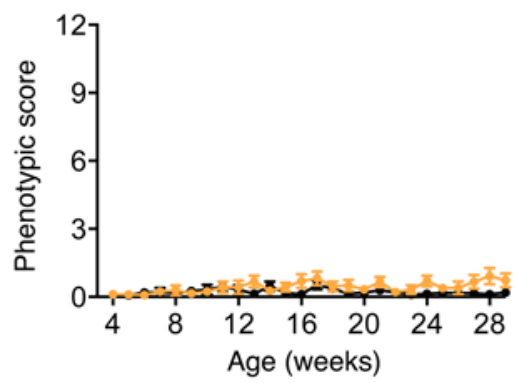

H

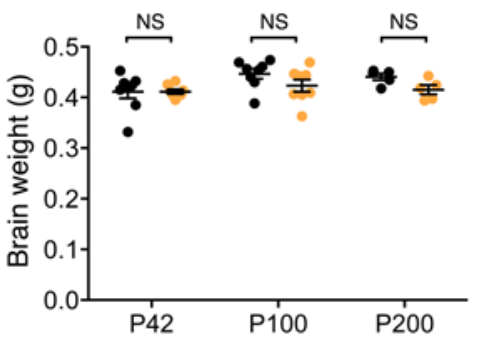

J

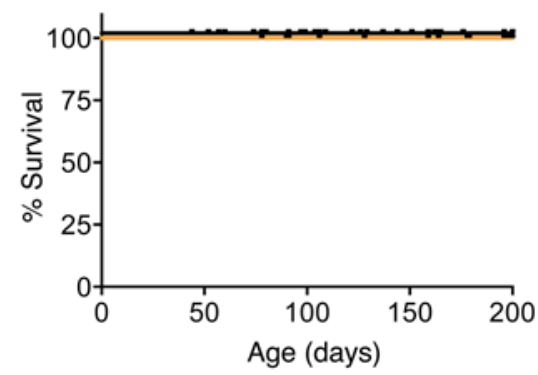

Figure 3. Generation and characterization of T158M-Tg mice. (A) Schematic of the construct used for transgenesis. Mecp2 T158M cDNA containing an N-terminal FLAG tag was placed under the control of the mouse prion promoter (Prp). (B) Western blot analysis of brain lysate from $2 \mathrm{~T} 158 \mathrm{M}-\mathrm{Tg}$ mouse lines (Tg2 and Tg3). FLAG-tagged Tg MeCP2 T158M (T158M-Tg) migrated slightly above endogenous MeCP2 levels. Graph shows quantification of T158M-Tg protein levels. Values were normalized to NeuN, and the results are shown as the percentage of endogenous MeCP2 ( $n=4 \mathrm{for}$ Tg2 and $n=5$ for Tg3). (C) Whole-brain nuclear lysate from T158M-Tg mice probed with a T158 site-specific antibody confirmed the presence of the mutation. (D) Sagittal sections stained for MeCP2 and FLAG show a similar distribution pattern for endogenous WT MeCP2 and Tg MeCP2 T158M throughout the brain. Scale bar: $500 \mu \mathrm{m}$. (E) Western blot analysis of Tg MeCP2 T158M protein levels in young (P20) and old (P200) T158M-Tg animals. (F) AntiFLAG immunoprecipitates from Mecp2 $2^{+/ y}$ T158M-Tg and Mecp2+/y whole brain were probed for the known MeCP2-interacting proteins SIN3A, TBLR1, and HDAC3. WB, Western blot. (C) Body weights of Mecp $2^{+/ y}$ male mice $\left(n=22\right.$, black) compared with Mecp2 ${ }^{+/ y} \mathrm{~T} 158 \mathrm{M}-\mathrm{Tg}$ male littermates $(n=15$, yellow) up to 28 weeks of age. (H) Brain weights at P42 ( $n=8$ for each genotype), P100 ( $n=8$ for each genotype), and P200 ( $n=5$ for each genotype). (I) Phenotypic scores ( $n=22$ for Mecp2 $2^{+/ y}$ mice and $n=15$ for Mecp2 $2^{+/ y}$ T158M-Tg mice) and (J) survival assessment $\left(n=21\right.$ for Mecp2 $2^{+/ y}$ mice and $n=35$ for Mecp2 $2^{+/ y} \mathrm{~T} 158 \mathrm{M}-\mathrm{Tg}$ mice) for Mecp2 ${ }^{+/ y} \mathrm{~T} 158 \mathrm{M}-\mathrm{Tg}$ males and WT littermates. In each assay, the findings for T158M-Tg mice were indistinguishable from those of WT mice. All error bars represent the mean \pm SEM. 
A

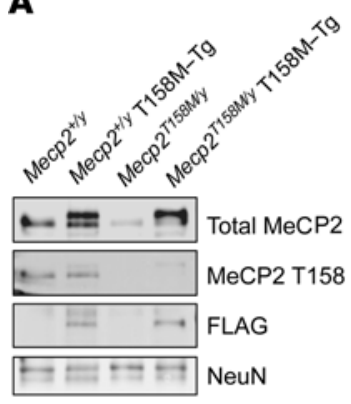

D

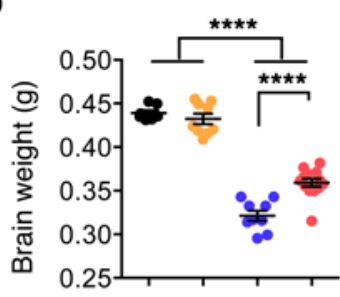

G

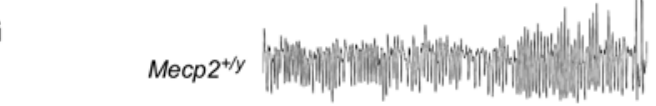

Mecp2 $2^{+/ y} \mathrm{~T} 158 \mathrm{M}-\mathrm{Tg}$

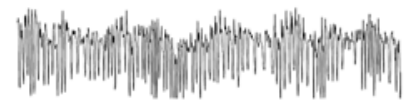

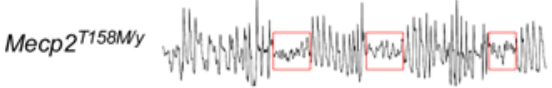

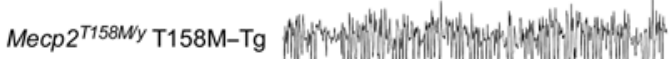

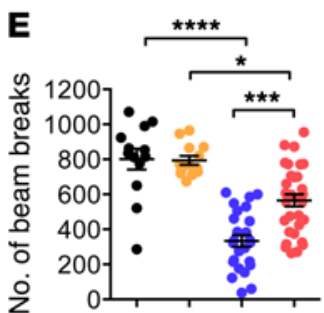

$-\mathrm{Mecp}^{+/ y}$

Mecp2 $2^{+/ y} \mathrm{~T} 158 \mathrm{M}-\mathrm{Tg}$

$\rightarrow$ Mecp2 $2^{T 158 M \mathrm{M}}$

$\rightarrow$ Mecp2 $2^{T 158 M y} \mathrm{~T} 158 \mathrm{M}-\mathrm{Tg}$
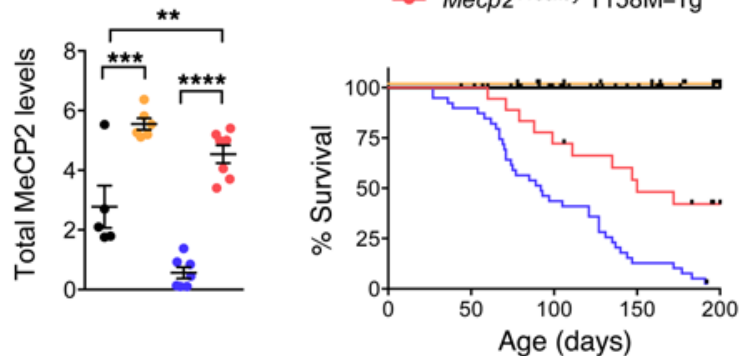

H

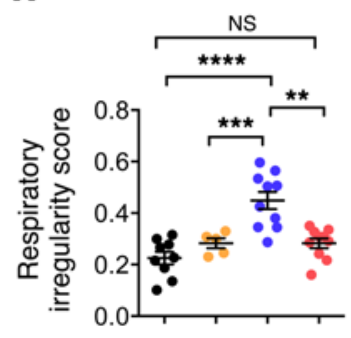

C
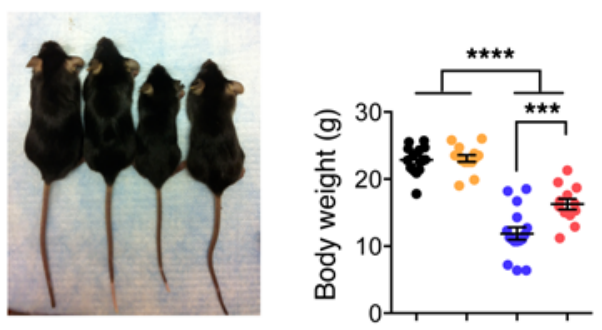

$\mathbf{F}$

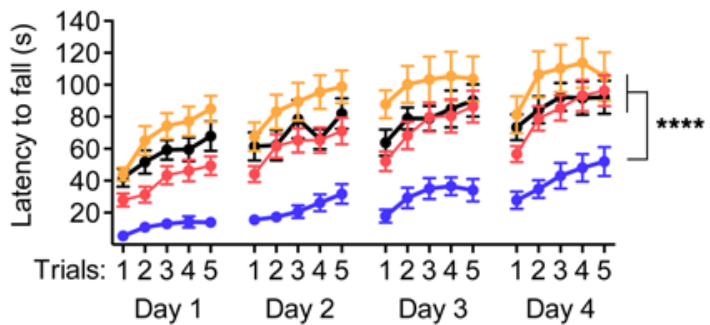

I

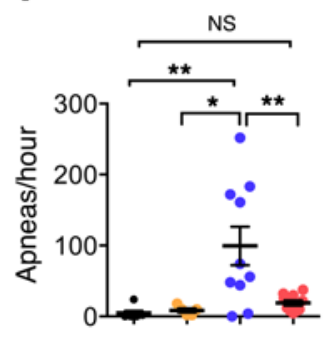

J
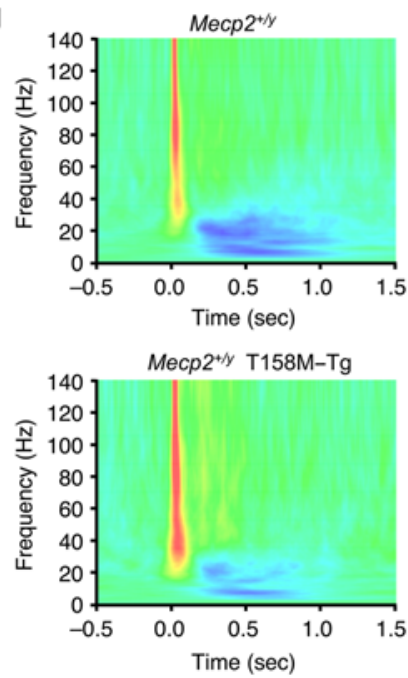

$\overline{2 \mathrm{sec}}$
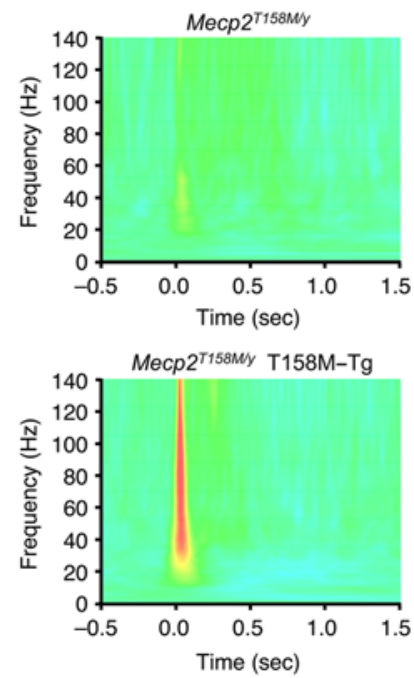
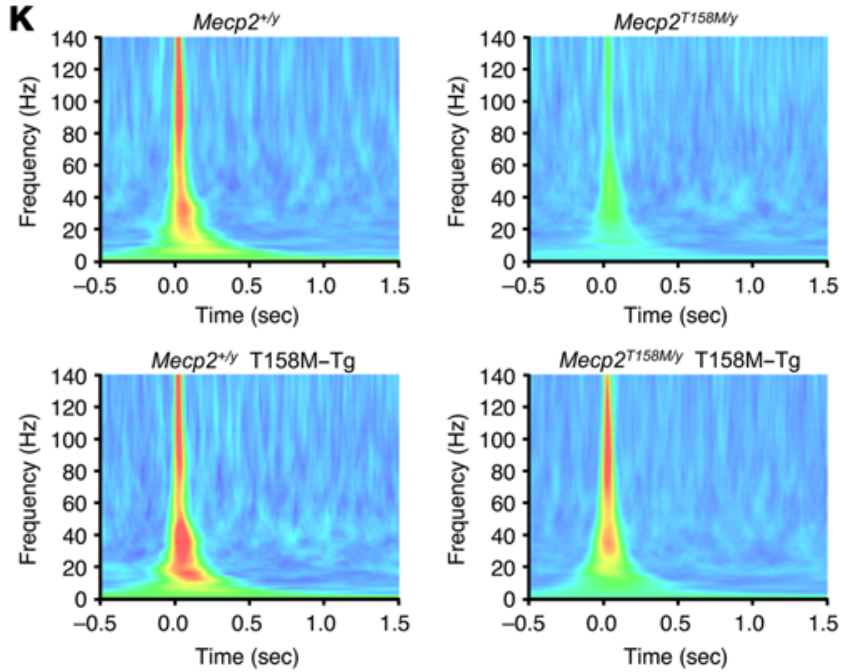
Figure 4. Tg elevation of MeCP2 T158M protein expression ameliorates behavioral deficits in male Mecp2 $2^{\mathrm{T158M} / \mathrm{y}}$ mice. (A) Western blot analysis of MeCP2 protein levels in brain nuclear lysate from male mice of all 4 genotypes at 12 weeks of age. Dot plot indicates the total amount of MeCP2 protein in Mecp2 $2^{+/ y}\left(n=5\right.$, black), Mecp2 ${ }^{+/ y} \operatorname{T158M-Tg~(~} n=6$, yellow), Mecp2 $2^{T 158 M / y}\left(n=7\right.$, blue), and Mecp2 $2^{T 158 M / y} \mathrm{~T} 158 \mathrm{M}-\mathrm{Tg}(n=7$, red) mice. MeCP2 levels were quantified using $\beta$-actin for normalization and are represented as the fold-change relative to Mecp2 $2^{T 158 M / y}$. (B) Kaplan-Meier survival curve ( $n=35,21,38$, and 18 per genotype) shows increased lifespan in Mecp2 ${ }^{T 158 M / y} \mathrm{~T} 158 \mathrm{M}-\mathrm{Tg}$ animals (median survival of $\sim 150$ days) relative to that of Mecp2 $2^{T 158 M / y}$ mice (median survival of $\sim 92$ days). Censored animals are indicated by a tick mark. (C) Body weights ( $n=14,14,16$, and 12) and (D) brain weights ( $n=7,9,9$, and 13) at 12 weeks were partially rescued in Mecp2 $2^{T 158 M / y} \mathrm{~T} 158 \mathrm{M}-\mathrm{Tg}$ animals. (E) The number of beam breaks in an open field assay $\left(n=13,13,25\right.$, and 31) was significantly increased in Mecp2 $2^{T 158 M / y}$ T158M-Tg animals compared with Mecp2 $2^{T 158 M / y}$ animals at 10 weeks of age. (F) Rotarod performance of 12-week-old animals ( $n=12,20,15$, and 29). The impaired performance of Mecp $2^{T 158 M / y}$ mice was rescued in Mecp2 $2^{T 158 M / y}$ T158M-Tg animals. ${ }^{* * *} P<0.0001$, by 2-way ANOVA. (C) Representative plethysmographic tracings (time bar: $2 \mathrm{~s}$ ). Red boxes delineate apneic episodes. (H) Respiratory irregularity scores and (I) number of apneas per hour $\left(n=9,5,10\right.$, and 10) at 8 weeks of age. Mecp $2^{T 158 M / y} \mathrm{~T} 158 \mathrm{M}-\mathrm{Tg}$ males had reduced respiratory irregularities and apneas relative to Mecp2 $2^{T 158 M / y}$ mice. Heatmaps indicating changes in (J) event-related power and (K) PLF in response to auditory stimulation in 10- to 14-week-old animals show rescue of information processing in Mecp2 $2^{T 158 M / y} \mathrm{~T} 158 \mathrm{M}-\mathrm{Tg}$ mice. Comparisons in $\mathbf{A}, \mathbf{C}, \mathbf{D}, \mathbf{E}, \mathbf{H}$, and I were done using 1-way ANOVA, followed by Tukey's post-hoc test. ${ }^{*} P<0.05$, ${ }^{* *} P<0.01,{ }^{* * *} P<0.001$, and ${ }^{* * * *} P<0.0001$. All error bars represent the mean \pm SEM.

mutant protein might ameliorate disease phenotypes. To test this, we obtained animals expressing elevated levels of MeCP2 T158M by breeding heterozygous Mecp $2^{T 158 M /+}$ females with $M e c p 2^{+/ y} \mathrm{~T} 158 \mathrm{M}-\mathrm{Tg}$ males. From these crosses, we produced male

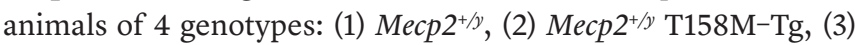
$M e c p 2^{T 158 M / y}$, and (4) Mecp2 $2^{T 158 M / y} \mathrm{~T} 158 \mathrm{M}-\mathrm{Tg}$. The latter genotype, Mecp $2^{T 158 \mathrm{M} / y} \mathrm{~T} 158 \mathrm{M}-\mathrm{Tg}$, represents animals with increased levels of MeCP2 T158M protein. Total MeCP2 protein expression in $M e c p 2^{T 158 M / y} \mathrm{~T} 158 \mathrm{M}-\mathrm{Tg}$ males was roughly twice that of WT $M e c p 2^{+/ y}$ animals, but approximately 4.5 -fold that of knockin $M e c p 2^{T 158 M / y}$ littermates (Figure 4A).

Following phenotypic characterization similar to that performed for Mecp $2^{T 158 M}$ mice, we found that $M e c p 2^{T 158 \mathrm{M} / \mathrm{y}} \mathrm{T} 158 \mathrm{M}-\mathrm{Tg}$ males appeared larger in size, were less hunched, and had smoother coats than did Mecp $2^{T 158 M / y}$ littermates (Supplemental Figure 4A). Notably, with a median survival of approximately 150 days, Mecp $2^{\text {T158M/y }} \mathrm{T} 158 \mathrm{M}-\mathrm{Tg}$ mice lived approximately twice as long as their Mecp2 $2^{T 158 M / y}$ littermates (Figure 4B). Mecp2 ${ }^{T 158 M / y} \mathrm{~T} 158 \mathrm{M}-\mathrm{Tg}$ mice also showed increased body weight and brain weight relative to Mecp $2^{T 158 M / y}$ animals (Figure $4, \mathrm{C}$ and $\mathrm{D}$ ).

To examine whether genetically increasing MeCP2 T158M expression ameliorates behavioral deficits in $M e c p 2^{T 158 M / y}$ mice, we subjected males of all 4 genotypes to a series of behavioral assessments. Given that motor dysfunction is a prominent feature of RTT, we measured locomotor activity using the open field assay. Consistent with our previous findings in Mecp2-null and MeCP2 T158A-knockin (Mecp2 ${ }^{T 158 A}$ ) mice (27), Mecp2 $2^{T 158 M}$ mice show significantly reduced locomotion compared with their WT littermates. In contrast, Mecp $2^{T 158 \mathrm{M} / \mathrm{y}} \mathrm{T} 158 \mathrm{M}-\mathrm{Tg}$ animals exhibited a marked improvement in locomotion, as measured by the total number of beam breaks (Figure 4E), distance traveled (Supple- mental Figure 4B), and average speed (Supplemental Figure 4C) in the open field arena relative to Mecp2 $2^{T 158 M / y}$ mice. Moreover, $M e c p 2^{T 158 M / y}$ mice performed significantly less well than did their WT littermates on the accelerating rotarod, consistent with previous reports (28). However, Mecp $2^{\text {T158M/y }} \mathrm{T} 158 \mathrm{M}-\mathrm{Tg}$ mice performed similarly to WT littermates, demonstrating a near-complete rescue in motor coordination and motor learning (Figure 4F).

Another key feature of RTT is respiratory abnormalities. We therefore examined respiratory function using whole-body plethysmography. Mecp $2^{T 158 M / \nu}$ mice experienced episodes of irregular breathing and had significantly increased incidences of apneas compared with $M e c p 2^{+/ y}$ and $M e c p 2^{+/ y} \mathrm{~T} 158 \mathrm{M}-\mathrm{Tg}$ mice. This is consistent with previous findings in Mecp2-null and Mecp2 $2^{\text {TI58A }}$ mice $(16,36,37)$ Notably, in Mecp $2^{T 158 M / y} \mathrm{~T} 158 \mathrm{M}-\mathrm{Tg}$ animals, we observed a rescue in overall breathing patterns and significantly reduced respiratory irregularity scores (Figures $4, \mathrm{G}$ and $\mathrm{H}$, and Supplemental Figure 4D). The number of apneas per hour, moreover, was reduced by approximately 5 -fold in Mecp $2^{T 158 M / y} \mathrm{~T} 158 \mathrm{M}-$ Tg mice compared with Mecp $2^{T 158 M / y}$ mice (Figure 4I).

Dysfunction in sensory information processing, measured as changes in visual or auditory event-related potentials (ERPs), has been observed in patients with RTT and in mouse models of RTT $(27,38-41)$. We therefore measured ERP responses following the presentation of sound stimulation in awake, freely mobile mice. Similar to Mecp2 $2^{T 158 A / y}$ mice, Mecp2 $2^{T 158 M / y}$ mice showed a significant reduction in event-related power and phase-locking factor (PLF) responses across all measured frequencies compared with $M e c p 2^{+/ y}$ mice, indicating impaired information processing (Figures $4, \mathrm{~J}$ and $\mathrm{K}$, and, Supplemental Figure 4E). Importantly, we found that $M e c p 2^{T 158 M / y} \mathrm{~T} 158 \mathrm{M}-\mathrm{Tg}$ mice showed a partial rescue of auditory-evoked power and PLF responses, particularly at higher frequencies (Figures 4, J and K, and Supplemental Figure 4E). The auditory-evoked power and PLF responses of $M e c p 2^{+/ y} \mathrm{~T} 158 \mathrm{M}-\mathrm{Tg}$ mice were not statistically different from those of $M e c p 2^{+/ y}$ mice (Supplemental Figure $4 \mathrm{~F}$ ). Taken together, our results show that augmenting the levels of MeCP2 T158M protein, despite its reduced affinity for DNA, substantially ameliorates motor, respiratory, and ERP deficits in male Mecp2 $2^{T 158 \mathrm{M} / \mathrm{y}}$ mice. In addition, Mecp2 $2^{T 158 \mathrm{M} / \mathrm{y}} \mathrm{T} 158 \mathrm{M}-\mathrm{Tg}$ mice behaved similarly to their WT littermates in all behavioral assessments, consistent with the notion that MeCP2 T158M shows no dominant-negative effects.

Assessing the dosage effect of MeCP2 T158M expression on phenotypic rescue. Given the strict dosage requirements for WT MeCP2, a critical question from a therapeutic standpoint is understanding the extent to which overexpression of MeCP2 T158M protein might introduce detrimental effects. To address this, we investigated the effects of doubling the expression levels of MeCP2 T158M by breeding the transgene to homozygosity, producing Mecp $2^{\text {T158M/y }} \mathrm{T} 158 \mathrm{M}-$ $\mathrm{Tg}$ homozygous male mice (Mecp $\left.2^{T 158 \mathrm{M} / \mathrm{J}} \mathrm{T} 158 \mathrm{M}-\mathrm{Tg} / \mathrm{T} 158 \mathrm{M}-\mathrm{Tg}\right)$. In Mecp $2^{T 158 \mathrm{M} / \mathrm{J}} \mathrm{T} 158 \mathrm{M}-\mathrm{Tg} / \mathrm{T} 158 \mathrm{M}-\mathrm{Tg}$ males, the total MeCP2 levels were approximately 3 -fold those of WT Mecp $2^{+/ y}$ male littermates, but approximately 8 -fold those of knockin Mecp $2^{T 158 M / y}$ male mice (Supplemental Figure 5A). We found that Mecp2 $2^{T 158 \mathrm{M} / \mathrm{y}} \mathrm{T} 158 \mathrm{M}-\mathrm{Tg} /$ T158M-Tg homozygous animals had body weights, brain weights, locomotor activity, and motor coordination and learning that were similar to those of $\mathrm{Mecp}^{\mathrm{T} 158 \mathrm{M} / \mathrm{y}} \mathrm{T} 158 \mathrm{M}-\mathrm{Tg}$ heterozygous animals 

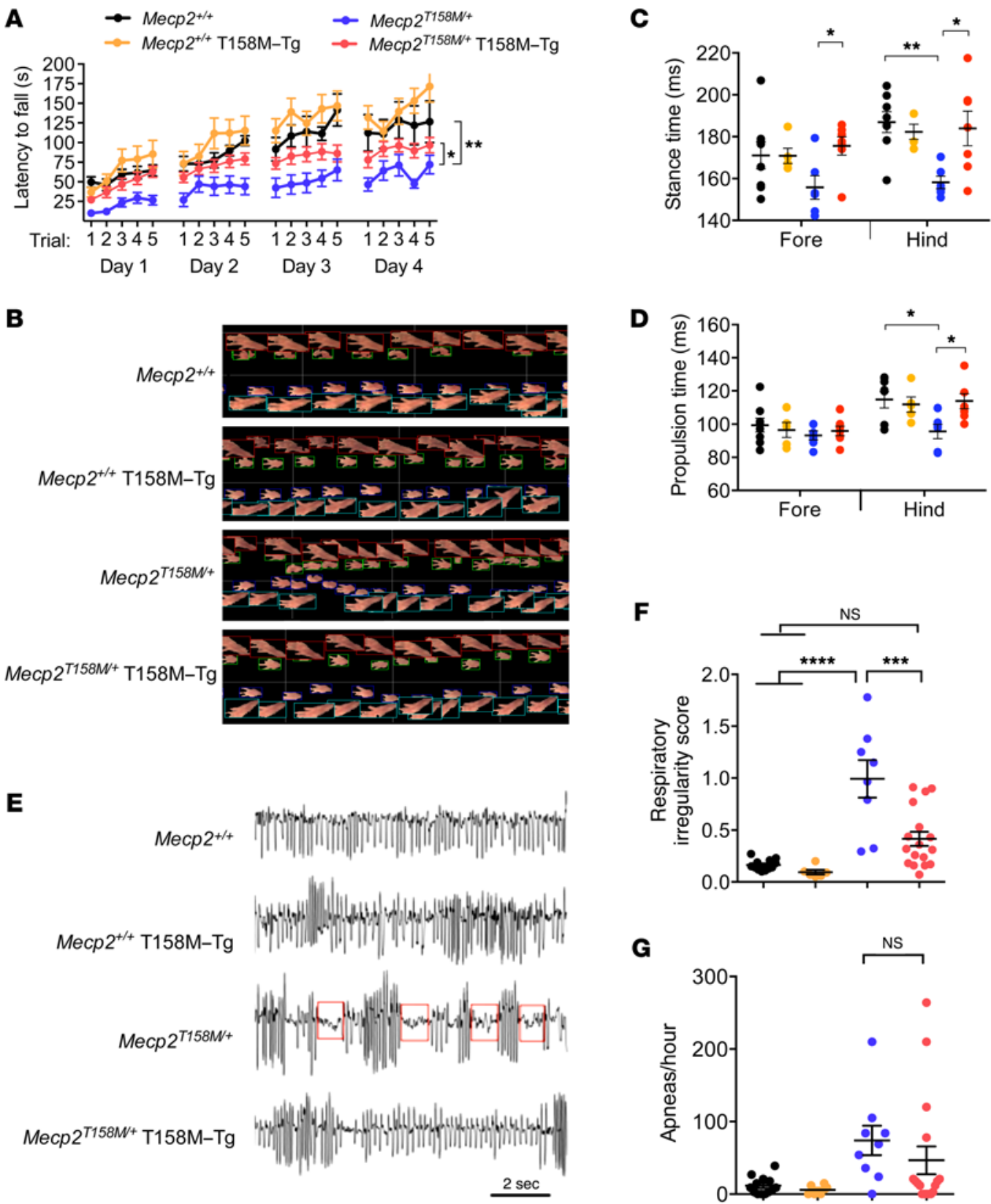

Figure 5. Genetically increasing the expression of MeCP2 T158M protein improves behavioral phenotypes in female Mecp2 $2^{\text {T158M/+ }}$ mice. (A) Latency to fall

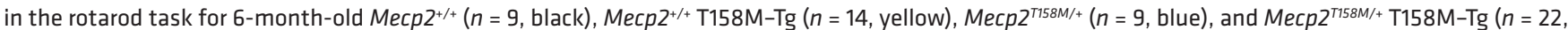
red) female mice. The deficit in Mecp $2^{T 158 M /+}$ mice was significantly more than that observed in Mecp2 $2^{+/+}\left({ }^{* *} P<0.01\right)$ and $M e c p 2^{T 158 M /+} T 158 \mathrm{M}-\mathrm{Tg}\left({ }^{*} P<0.05\right.$, by 2-way ANOVA) mice. (B) Representative images of paw placements on the treadmill surface for 7-month-old female mice of each genotype. (C) Stance and (D) propulsion times for the fore- and hind paws ( $n=8,5,6$, and 7 per genotype). Mecp $2^{T 158 M /+}$ female mice had reduced stance and propulsion times in the hind paws. These deficits were rescued in Mecp2 ${ }^{\mathrm{T} 158 \mathrm{M} /+} \mathrm{T} 158 \mathrm{M}-\mathrm{Tg}$ littermates. (E) Representative plethysmographic tracings (time bar: $2 \mathrm{~s}$ ). Red boxes delineate apneic episodes. (F) Average respiratory irregularity scores $(n=12,6,8$, and 17 per genotype) and (G) number of apneas per hour $(n=13,6,9$, and 17 per genotype) for 6 - to 9-month-old female mice. Mecp $2^{T 158 M /+} \mathrm{T} 158 \mathrm{M}$-Tg females had significantly reduced respiratory irregularity scores relative to Mecp $2^{T 158 M /+}$ mice. Comparisons in $\mathbf{C}, \mathbf{D}, \mathbf{F}$, and $\mathbf{G}$ were done using 1-way ANOVA, followed by Tukey's post-hoc test. ${ }^{*} P<0.05,{ }^{* *} P<0.01$, ${ }^{* * *} P<0.001$, and ${ }^{* * * *} P<0.0001$. All error bars represent the $\pm \mathrm{SEM}$.

(Supplemental Figure 5, B-E). Thus, low and high MeCP2 T158M transgene expression confers similar behavioral improvements in male mice. Further, these results indicate that an increased MeCP2 T158M dosage is not deleterious and that MeCP2 T158M expression levels do not need to be as tightly controlled as do those of WT MeCP2. Taken together, these data demonstrate that Tg elevation of MeCP2 T158M expression, even at levels approximately 3-fold higher than WT MeCP2 levels, markedly improves behavioral performance in $M e c p 2^{T 158 M / y}$ male mice.

Increasing MeCP2 T158M expression ameliorates behavioral impairments in Mecp $2^{T 158 M /+}$ female mice. Since RTT is primarily a female disorder, we next examined whether genetic elevation of 
MeCP2 T158M expression also ameliorates RTT-like phenotypes in female mice. We first subjected female mice to the accelerating rotarod task. Consistent with findings in heterozygous Mecp2-null females $(36,42,43)$, Mecp $2^{T 158 M /+}$ females showed a significantly reduced latency to fall from the rotarod compared with WT $\mathrm{Mecp2}^{+/+}$littermates (Figure 5). By contrast, the rotarod performance of $M e c p 2^{\text {T158M/+ }} \mathrm{T} 158 \mathrm{M}-\mathrm{Tg}$ female mice was nearly indistinguishable from that of WT Mecp $2^{+/+}$mice, indicating a full rescue of motor coordination and motor skill learning (Figure 5A). Given that gait abnormalities are a prominent feature of RTT, we next analyzed gait behavior in our female mice using the TreadScan system, which uses a transparent treadmill belt and high-speed camera to assess gait characteristics in rodent models. The system has been used previously to evaluate RTT-associated motor dysfunction (44). We found that Mecp $2^{T 158 M /+}$ female mice showed a more uncoordinated gait overall relative to mice of the other genotypes (Figure $5 \mathrm{~B}$ ) and had significantly reduced hind paw stance times, which is the duration the foot is in contact with the treadmill. This abnormality was rescued in Mecp $2^{\text {T158M/+ }} \mathrm{T} 158 \mathrm{M}-\mathrm{Tg}$ animals (Figure 5C). Mecp $2^{T 158 M /+}$ female mice, moreover, had significantly reduced propulsion times in the hind paws relative to both Mecp2 $^{+/+}$and Mecp2 $^{+/+}$T158M-Tg females, a phenotype that was also rescued in Mecp $2^{\mathrm{T158M} /+} \mathrm{T} 158 \mathrm{M}-\mathrm{Tg}$ mice (Figure 5D).

Given the characteristic breathing abnormalities in female patients with RTT and in mouse models of RTT, we next evaluated respiratory function using whole-body plethysmography. Similar to previous findings in heterozygous Mecp2-null female mice (16, 36), Mecp $2^{T 158 M /+}$ females had irregular breathing patterns and significantly increased breathing irregularity scores compared with WT $M e c p 2^{+/+}$mice. Notably, the irregularity scores of $M e c p 2^{\text {T158M/+ }}$ T158M-Tg females were significantly reduced compared with those for $M e c p 2^{T 158 M /+}$ mice, indicating a significantly improved breathing pattern (Figure 5, E and F). Additionally, Mecp2 $2^{T 158 M /+}$ T158M-Tg female mice had reduced numbers of apneas per hour compared with Mecp $2^{\text {T158M/+ }}$ females (Figure 5G). Though the latter assessment was not statistically significant because of 2 severe cases, the majority of Mecp $2^{T 158 M /+} \mathrm{T} 158 \mathrm{M}-\mathrm{Tg}$ female mice did not have apneas (Figure 5G). Together, these data demonstrate that increasing MeCP2 T158M protein expression also ameliorates a number of symptoms associated with RTT in female mouse models.

Genetic elevation of MeCP2 T158M expression in Mecp $2^{T 158 M / y}$ mice increases DNA binding. Given our findings in cultured N2a cells, we next tested the possibility that phenotypic amelioration might be mediated by increased binding of MeCP2 T158M to DNA in vivo. We thus performed IHC using anti-MeCP2 antibodies to assess the in vivo binding pattern of MeCP2 in all 4 genotypes of male mice (Figure 6A). Consistent with our previous findings, WT MeCP2 showed a punctate pattern of staining, reflecting the binding of MeCP2 to heterochromatic foci, while MeCP2 T158M showed a diffuse pattern, with no observed puncta. In contrast, Mecp $2^{\text {T158M/y }} \mathrm{T} 158 \mathrm{M}-\mathrm{Tg}$ mice showed increased MeCP2 staining intensity compared with Mecp $2^{T 158 M / y}$ animals, consistent with elevated MeCP2 T158M protein expression. In addition, we observed an increased incidence of MeCP2 colocalization with heterochromatic foci in $M e c p 2^{T 158 M / y} \mathrm{~T} 158 \mathrm{M}-\mathrm{Tg}$ animals compared with Mecp2 $2^{T 158 M / y}$ littermates, supporting the idea that MeCP2 T158M binds to DNA in an expression level-dependent manner (Figure
6A). These results differ slightly from those in N2a cells, in which increased puncta formation in the high-expressing FLAG-T158MDendra2 cell line was observed. The difference may lie in the fact that N2a cells are a tumor-derived cell line made up of phenotypically immature neurons that may not accurately recapitulate neuronal features in the adult brain.

The majority of WT MeCP2 is tightly bound to chromatin in the nucleus and is extracted under high ionic conditions or nuclease treatment. By contrast, mutation at $\mathrm{T} 158$ results in extraction of $\mathrm{MeCP} 2$ at lower salt concentrations because of the reduced affinity of MeCP2 for methylated DNA (27). To compare the subnuclear distributions of MeCP2 in Mecp2 $2^{+/ y}, M e c p 2^{T 158 M / y}$, and $M e c p 2^{T 158 M / y}$ $\mathrm{T} 158 \mathrm{M}-\mathrm{Tg}$ animals, we performed subnuclear fractionation experiments in cortical nuclei (Figure 6B). As expected, the bulk of WT $\mathrm{MeCP} 2$ is found in the chromatin fraction (Chr), with relatively little present in the nucleosolic fraction (Nuc). In Mecp $2^{T 158 M / y}$ animals, as shown previously, the overall levels of MeCP2 T158M were reduced, and MeCP2 T158M localization was predominantly nucleosolic, not chromatin bound. Similarly, in Mecp $2^{T 158 M / y} \mathrm{~T} 158 \mathrm{M}-\mathrm{Tg}$ animals, the majority of mutant protein was present in the nucleosolic fraction as opposed to the chromatin fraction, in agreement with the relatively diffuse pattern observed with IHC (Figure 6A). However, the levels of MeCP2 T158M in both the nucleosolic and chromatin-bound fractions were increased relative to their respective fractions in $M e c p 2^{T 158 M / y}$ animals (Figure 6B). Thus, the overall elevated expression of MeCP2 T158M protein comprises both a nucleosolic pool and, to a lesser extent, a chromatin-bound pool.

To evaluate whether the increased chromatin-bound MeCP2 $\mathrm{T} 158 \mathrm{M}$ reflects increased $\mathrm{MeCP} 2$ binding to specific genomic loci, we next performed MeCP2 ChIP-quantitative PCR (ChIP-qPCR) on cortical tissue from $M e c p 2^{+/ y}, M e c p 2^{T 158 M / y}$, and $M e c p 2^{T 158 M / y}$ T158M-Tg animals (Figure 6C). In Mecp $2^{+/ y}$ animals, MeCP2 bound to known target loci within the Sst, Bdnf, Crh, and Nr4a1 genes, the promoters of LINE-1 and IAP repeat elements, and, to a lesser extent, at the Gapdh promoter $(29,30,45)$. By contrast, in $M e c p 2^{T 158 M / y}$ animals, the binding of MeCP2 T158M to each locus was markedly reduced. Notably, in Mecp $2^{T 158 M / y} \mathrm{~T} 158 \mathrm{M}-\mathrm{Tg}$ animals, the binding of MeCP2 T158M was elevated overall relative to that seen in Mecp $2^{T 158 M / y}$ animals at each MeCP2 target site (Figure 6C). Together, these data demonstrate that increasing MeCP2 $\mathrm{T} 158 \mathrm{M}$ protein expression in vivo overcomes the DNA-binding defect associated with this mutant, leading to amelioration of RTT-like phenotypes.

Proteasome inhibition increases MeCP2 T158M protein levels. Having found that genetic overexpression of MeCP2 T158M rescues DNA binding and RTT-like phenotypes in mice, we next sought to increase MeCP2 T158M levels via a pharmacological approach. We first sought to identify the molecular pathway responsible for MeCP2 T158M degradation. We therefore cultured N2a FLAG-MeCP2-Dendra2 clonal cell lines and treated them with various inhibitors of protein degradation pathways, including calpeptin (calpain inhibitor), 3-methyladenine (3-MA; autophagy inhibitor), ammonium chloride $\left(\mathrm{NH}_{4} \mathrm{Cl}\right.$; lysosome inhibitor), and MG132 (proteasome inhibitor). We found that MG132 increased FLAG-MeCP2-Dendra2 levels to the greatest extent (Figure 6D), indicating that the ubiquitin/proteasome pathway is the primary pathway by which MeCP2 is degraded. While we found a detect- 
A

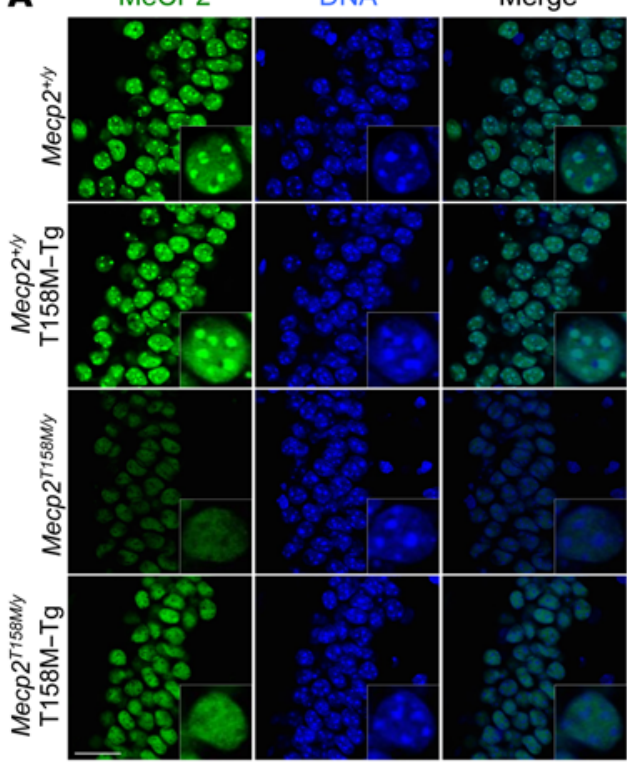

D
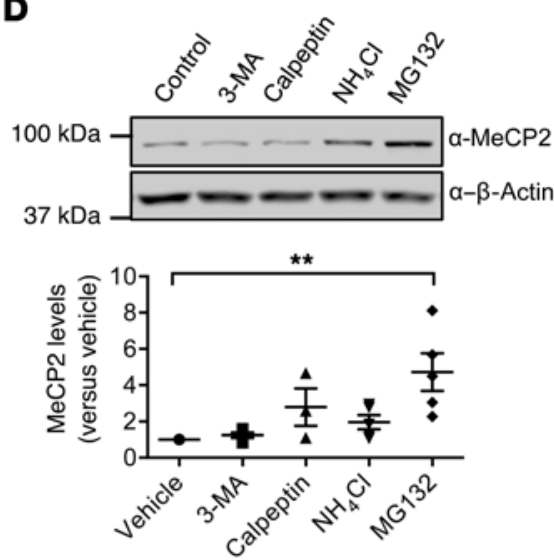

F $\quad$ FLAG-T158M-Dendra2

MG132 $(\mu \mathrm{M}): 0 \begin{array}{llllll}0 & 5 & 10 & 15 & 20 & 40\end{array}$
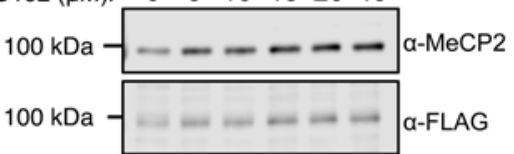

$37 \mathrm{kDa}-----\infty \mathrm{\alpha}-\beta$-Actin

$37 \mathrm{kD}$
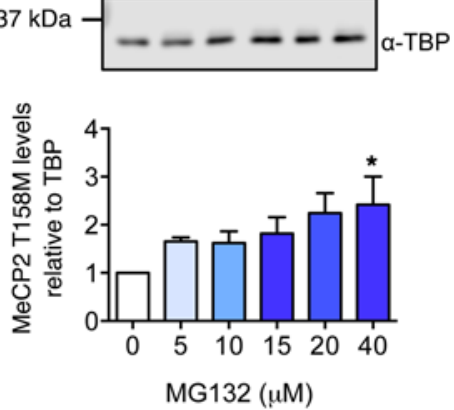

B
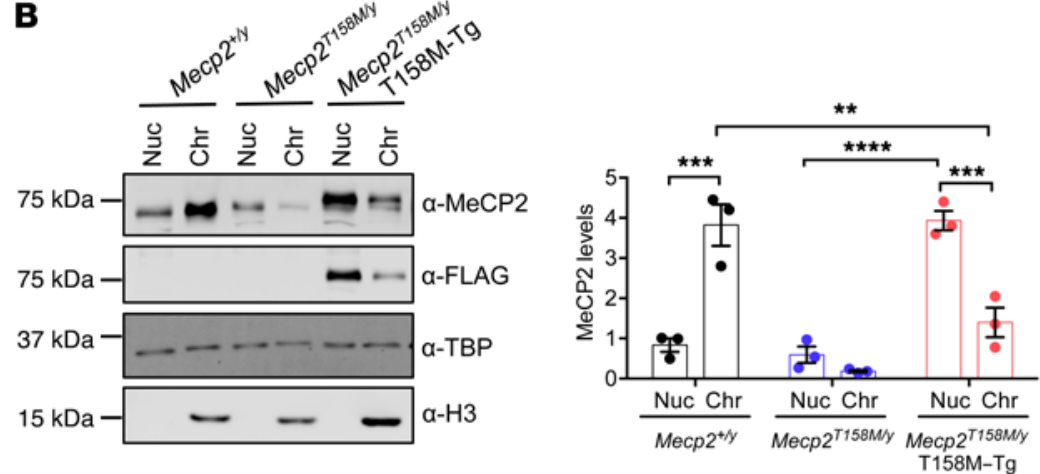

C

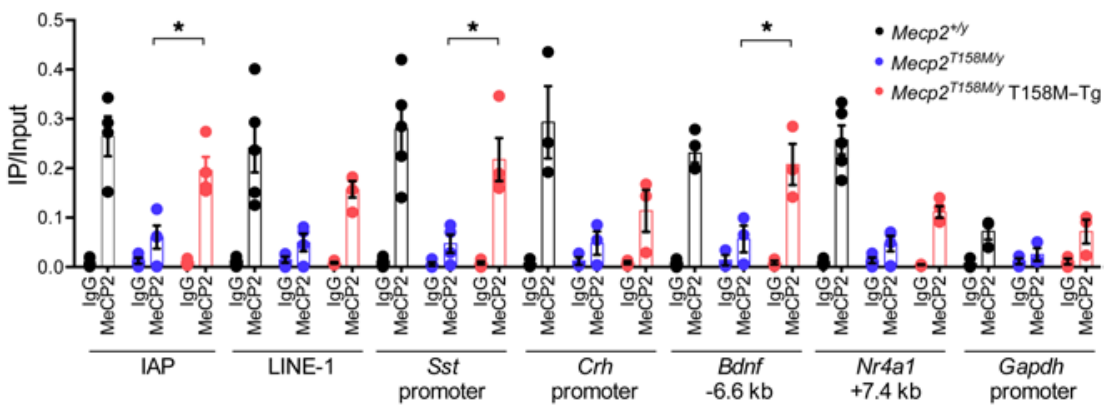

E
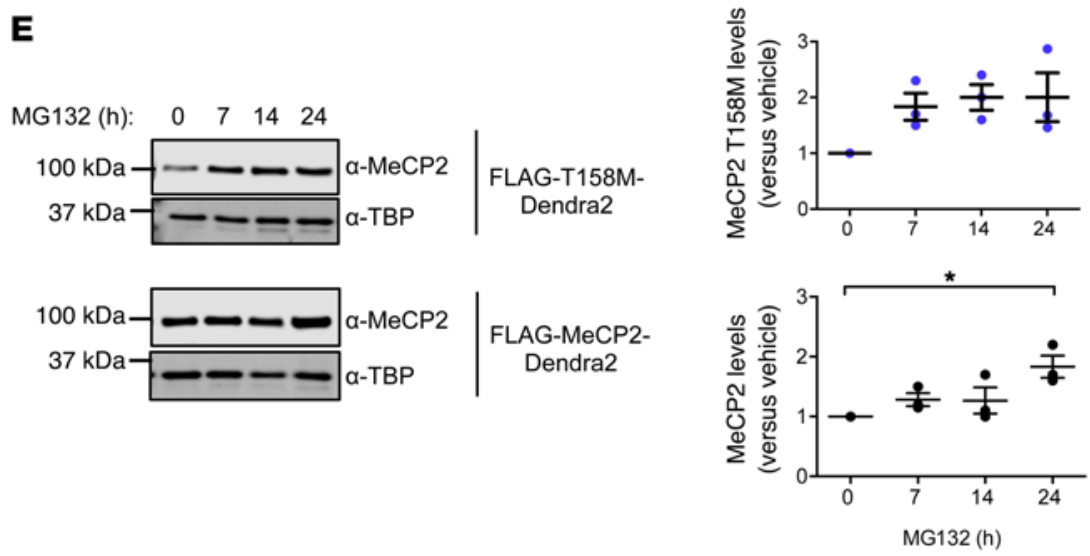

G
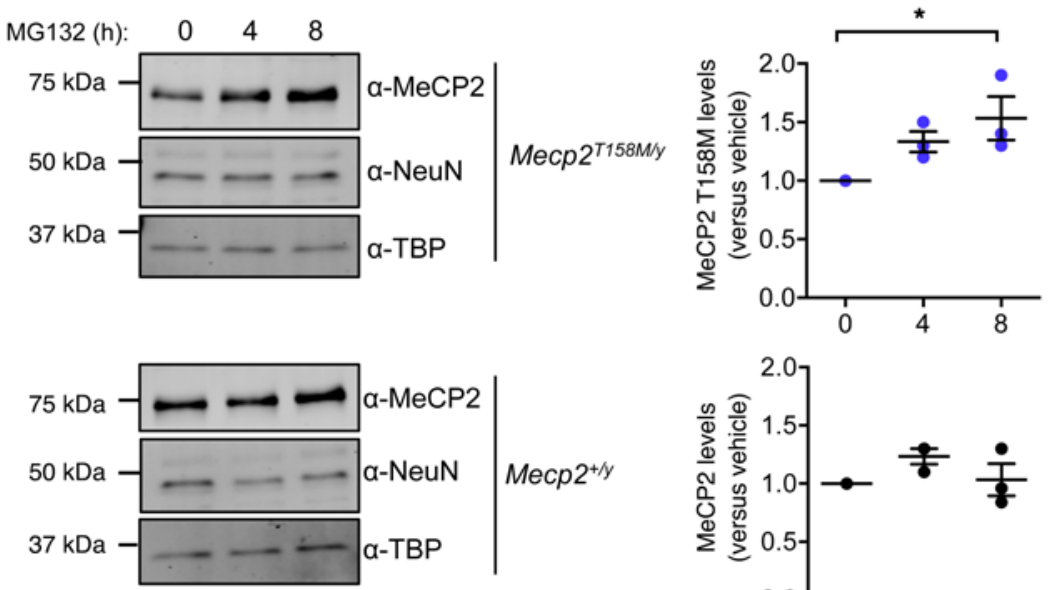


\section{Figure 6. Tg elevation of MeCP2 T158M expression increases DNA}

binding. (A) IHC for MeCP2 in brain sections from animals of the indicated genotype at 12 weeks. Scale bar: $20 \mu \mathrm{m}$. Original magnification: $\times 63$ with digital zoom. (B) Western blot of nucleosolic (Nuc) and chromatin-enriched (Chr) proteins in cortices from Mecp2 $2^{+/ y}, \mathrm{Mecp}^{T 158 \mathrm{M} / \mathrm{y}}$, and Mecp2 $2^{T 158 \mathrm{M} / \mathrm{y}}$ T158M-Tg animals at 12 weeks. Graph shows the quantification of MeCP2 levels in nucleosolic and chromatin fractions normalized to TBP. Values are represented as the fold-change relative to nucleosolic MeCP2 from Mecp $2^{+/ y}$ animals ( $n=3$ per genotype). ${ }^{* *} P<0.01,{ }^{* * *} P<0.001$, and ${ }^{* * *} P<0.0001$, by 2 -way ANOVA, followed by Sidak's multiple comparisons test. (C) MeCP2 ChIP-qPCR in cortices from 12-week-old Mecp2 ${ }^{+/ y}$, Mecp2 $2^{T 158 M / y}$, and Mecp2 $2^{T 158 M / y} \mathrm{T158M}$-Tg animals at the indicated loci revealed significantly increased MeCP2 binding in Mecp2 $2^{\mathrm{T15} 8 \mathrm{M} / \mathrm{y}} \mathrm{T} 158 \mathrm{M}-\mathrm{Tg}$ animals at several loci ( $n=3-5$ biological replicates). ${ }^{*} P<0.05$, by 1 -way ANOVA, followed by Tukey's post-hoc test. (D) FLAG-MeCP2-Dendra2 protein levels following treatment with inhibitors of protein degradation pathways. MG132 increased the levels of MeCP2 to the greatest extent. Values were normalized to $\beta$-actin and are represented as the fold change relative to vehicle-treated cells ( $n=3-5$ per condition). (E) Time course of MG132 treatment in FLAG-T158M-Dendra2 and FLAG-MeCP2-Dendra2 N2a cell lines ( $n=3$ biological replicates). (F) FLAG-T158M-Dendra2 levels following treatment with increasing concentrations of MG132 ( $n=3$ per condition). (C) PO plus 3-DIV cortical cultures from Mecp2 $2^{+/ y}$ and $M e c p 2^{T 158 M / y}$ animals treated with vehicle or $20 \mu \mathrm{M}$ MG132 for 4 and 8 hours $(n=3$ per genotype). Quantifications in E-G were performed by normalizing to the TBP loading control; values are shown as the fold-change relative to to. Comparisons in $\mathbf{D}-\mathbf{G}$ were done using 1-way ANOVA, followed by Dunnett's multiple comparisons test. ${ }^{*} P<0.05$ and ${ }^{* *} P<0.01$. All error bars represent the mean \pm SEM.

able increase in WT FLAG-MeCP2-Dendra2 levels after 24 hours of MG132 treatment, elevated FLAG-T158M-Dendra2 levels were detectable after 7 hours (Figure 6E), reflecting the reduced stability of MeCP2 T158M. Notably, treatment with increasing concentrations of MG132 led to a dose-dependent increase in FLAG-T158M-Dendra2 levels (Figure 6F). We also observed a dose-dependent increase in MeCP2 T158M levels with the more specific proteasome inhibitor lactacystin (Supplemental Figure 6).

To examine whether endogenous MeCP2 T158M is also stabilized by proteasome inhibition, we cultured cortical neurons from $M e c p 2^{T 158 M / y}$ and $M e c p 2^{+/ y}$ animals and treated them with MG132 for 4 and 8 hours. We found that MeCP2 T158M levels significantly increased after 8 hours, while WT MeCP2 levels remained relatively unchanged, consistent with the increased stability of the WT protein (Figure 6G). Thus, MeCP2 T158M protein can be stabilized pharmacologically with proteasome inhibitors, revealing the ubiquitin/proteasome pathway as a pathway to target in the treatment of RTT.

\section{Discussion}

A major advance in the RTT field is the finding that restoration of endogenous MeCP2 expression in symptomatic Mecp2-null mice leads to reversal of RTT-like phenotypes, supporting the possibility that RTT is a treatable condition. Despite this remarkable achievement, determining how to restore normal MeCP2 function in RTT patients remains a challenge. In this study, we found that the binding of MeCP2 T158M to methylated DNA is at least in part dependent on its protein expression level. This prompted us to test whether increasing the expression of the mutant protein might overcome to a degree the reduced affinity of this mutant for DNA and partially restore $\mathrm{MeCP} 2$ function. Indeed, Tg elevation of
MeCP2 T158M expression increased DNA binding and improved behavioral phenotypes and the overall health of both male and female $M e c p 2^{T 158 M}$ mice. To our knowledge, these findings are the first to implicate mutant $\mathrm{MeCP} 2$ protein expression level as a contributing factor in RTT pathogenesis and reveal the targeting of $\mathrm{MeCP} 2$ protein expression or stability as a potential therapeutic approach for the treatment of RTT.

Several therapeutic approaches for treating RTT are being developed (43, 46-48), with adeno-associated virus-mediated (AAV-mediated) gene therapy at the forefront (16). A longstanding theoretical concern for gene therapy is that patient-associated mutations may exert dominant-negative effects and that this would limit the function of the virally delivered WT copy. Given that patients with RTT are mosaic females, in which approximately half of the cells express the WT Mecp2 allele, while the other half express the mutant allele, reactivation of the WT copy of Mecp 2 on the inactive $\mathrm{X}$ chromosome (Xi) has been proposed as another potential strategy to treat the disorder. However, as with gene therapy approaches, it is also critical to determine in this context whether RTT-causing mutations act in a dominant-negative fashion, since both WT and mutant copies will be expressed in the same cell upon reactivation of the Xi. Thus, to determine the extent of a potential therapeutic benefit using these strategies, a necessary first step is to assess whether RTT-associated mutations have dominant-negative effects.

Previous studies also reported that $\mathrm{MeCP} 2$ expression levels must be precisely controlled, with even a 1-fold increase in WT levels resulting in neurological phenotypes (17-22). We found that male and female T158M-Tg mice showed no behavioral abnormalities or reduced longevity, suggesting that Tg T158M protein produces no dominant-negative effects on endogenous WT MeCP2. The T158M allele may thus be considered a partial loss of function. Thus, our data provide support for gene therapy approaches in patients with mutations at T158. Our data also support the idea that reactivation of the silenced Mecp2 allele, either WT or $\mathrm{T} 158 \mathrm{M}$, would be beneficial. Importantly, we observed no deleterious effects on Mecp2 $2^{T 158 M / y}$ mice expressing MeCP2 levels approximately 3-fold higher than those in WT mice; in fact, the level of phenotypic improvement was similar in Mecp $2^{\text {T158M/y }}$ animals with 1 or 2 copies of the Mecp2 T158M transgene. This implies a potentially broader dosage window for therapeutic efficacy than expected from previous studies, an encouraging observation for future pharmacologic interventions. We are aware that our Mecp2 $2^{T 158 M / y}$ T158M-Tg mice did not exhibit a complete rescue of RTT-like phenotypes. This could be due to the fact that $\mathrm{T} 158 \mathrm{M}-\mathrm{Tg}$ protein is only expressed in neuronal cells in the CNS, but is missing astrocytes and other non-neuronal cells $(49,50)$. In addition, MeCP2 T158M, even though expressed at higher levels than WT MeCP2, may not fully function as a WT protein.

Interestingly, we found that the average respiratory irregularity scores of $M e c p 2^{T 158 M /+}$ females were higher than those of Mecp $2^{T 158 M / y}$ males and those previously reported for heterozygous Mecp $2^{-/+}$females $(16,51)$. Female patients with RTT are known to exhibit significant autonomic instability that manifests, in part, as ventilatory timing irregularities, including breath holding, apneas, tachypnea, and hyperpneas (52). Mecp $2^{T 158 M /+}$ female mice in the present study showed marked respiratory variability, with greater 
periods of tachypnea fluctuating with apneas, resulting in higher irregularity scores than previously observed in $\mathrm{Mecp}^{-/+}$female mice (51). The mean irregularity scores of WT and $\mathrm{T} 158 \mathrm{M}-\mathrm{Tg}$ males and females are similar to scores reported previously for WT animals $(37,51,53)$, while $M e c p 2^{T 158 M / y}$ male mice have irregularity scores similar to those reported for $M e c p 2^{-/ y}$ and $M e c p 2^{R 168 X / y}$ males $(51,53)$. An important difference, we believe, lies in the ages of the female and male mice used in these studies. In our study, the Mecp $2^{T 158 M / y}$ males were analyzed for breathing abnormalities at approximately 2 months of age, while the Mecp $2^{T 158 M /+}$ females were measured at 6 to 9 months of age. The $M e c p 2^{-/+}$females analyzed previously were between 9.8 and 14.5 months of age (51). Given previous findings that abnormal breathing patterns improve with advanced age in both patients with RTT and mouse models of RTT $(37,54)$, the severity of respiratory phenotypes, like other RTT-like behavioral abnormalities, greatly depends on the age at the time of measurement. Other factors, such as the particular $\mathrm{MeCP} 2$ mutation and the pattern of $\mathrm{X}$-chromosome inactivation (XCI), also play important roles in female phenotypic severity.

Our findings also point to several new clinically feasible approaches to potentially restore $\mathrm{MeCP} 2$ function, thereby tackling the root cause of the disorder. We show that mutation at T158 destabilizes MeCP2 protein, resulting in its degradation, and that elevating the levels of mutant protein reverses numerous disease phenotypes. One potential strategy to increase MeCP2 T158M protein levels is to selectively inhibit its degradation. Here, we have identified the ubiquitin/proteasome pathway as the one by which MeCP2 T158M is degraded, highlighting this pathway as a potential drug target. Although it is conceivable that treatment with proteasome inhibitors may provide clinical benefit to patients, the lack of brain-penetrant proteasome inhibitors could complicate delivery efforts. Proteasome inhibitors, moreover, target many cellular proteins and would exert many widespread, nonspecific effects. Future work directed at identifying the specific proteins involved in MeCP2 T158M turnover, such as the MeCP2 T158M-specific E3 ubiquitin ligase, is necessary to further refine potential therapeutic targets. High-throughput compound screens, moreover, can be used to identify compounds that stabilize MeCP2 T158M protein. Aside from inhibiting degradation, an alternative therapeutic strategy is to increase Mecp2 T158M expression at either the transcriptional or translational level. Thus, utilizing CRISPR activation (CRISPRa) technology and AAV delivery vehicles to target transcriptional activators to the endogenous $M e c p 2^{T 158 M}$ allele to boost transcription might be a feasible in vivo approach.

Several hundred patient-associated MeCP2 mutations spanning the entire protein have been identified. These mutations include missense, frameshift, nonsense, and splice-site mutations (RettBASE, http://mecp2.chw.edu.au). Notably, missense mutations in the MBD constitute approximately $25 \%$ of all RTT cases, underscoring the importance of this domain for proper MeCP2 function. We have recently found that another frequent RTTassociated missense mutation in the MBD, MeCP2 R106W, also has reduced protein stability (B.S. Johnson, unpublished observations), suggesting that mutations in the MBD might destabilize MeCP2 in a general fashion. If this is the case, therapies that stabilize or increase the expression of mutant MeCP2 have the potential to benefit many patients, not just those with MeCP2 T158M mutations. Thus, we believe our findings represent the first step toward the development of a tailored therapeutic approach for individuals bearing missense mutations in the MBD and provide support for the development of personalized medicine to treat RTT and other genetic diseases.

\section{Methods}

\section{Generation of Mecp2 ${ }^{T 158 M}$ - and T158M-Tg mice}

The targeting construct used to generate $M e c p 2^{T 158 M}$ mice was similar to that described previously (27). Threonine 158 was mutated to methionine using the QuikChange Site-directed Mutagenesis Kit (Agilent Technologies). The locus was engineered to contain a 23-amino acid Tavi affinity tag that contains a biotinylation consensus motif, allowing for BirA-dependent biotinylation of MeCP2. The targeting construct was linearized using NotI and electroporated into sv129 mouse embryonic stem (ES) cells. Correctly targeted clones were identified by PCR screening, verified by Southern blotting, and injected into C57BL/6 blastocysts and subsequently implanted into pseudopregnant females. Resulting chimeras were mated with C57BL/6 EIIa-Cre for embryonic deletion of the neomycin (Neo) cassette and subsequently backcrossed with C57BL/6 mice for at least 5 generations. Mecp $2^{T 158 M}$ mice were obtained from The Jackson Laboratory (stock no. 029642).

To generate FLAG-Mecp $2^{\text {T158MTg }}$ (T158M-Tg), Mecp2-e1 cDNA from mice was mutagenized to incorporate the T158M mutation and engineered to contain an N-terminal FLAG tag. The resulting cDNA was subcloned into the XhoI site of the MoPrP.Xho vector (32), linearized with NotI, and gel purified. The construct was microinjected into the pronuclei of fertilized C57BL/6 eggs and implanted into pseudopregnant female mice. Screening for Tg founders was performed by PCR genotyping using the primers described below. Tg mice were maintained on a C57BL/6 background.

\section{Animal husbandry}

$M e c p 2^{T 158 M / y}$ mice were PCR genotyped using the following primers that span the Tavi tag: forward, 5'-CACCCCGAAGCCACGAAACTC-3'; reverse, 5'-TAAGACTCAGCCTATGGTCGCC-3'. Tg mice were genotyped using primers (forward, $5^{\prime}$-GATCTGCTGGAAAGTATGATGTATATTTG-3'; reverse, 5'-CAACAAGTTTCCCAGGGCTC-3') that span an MeCP2 intron, giving rise to 2 bands ( 320 and $808 \mathrm{bp})$ in $\mathrm{Tg}$ animals and 1 band ( $808 \mathrm{bp}$ ) in WT animals. Male T158M-Tg mice were crossed with $M e c p 2^{\text {T158M/+ }}$ or Mecp $2^{\text {T158M/+ }} \mathrm{T} 158 \mathrm{M}-\mathrm{Tg}$ females to generate Mecp $2^{\text {T158M/y }} \mathrm{T} 158 \mathrm{M}-\mathrm{Tg}$ or $\mathrm{Mecp}^{2^{T 158 M} /+} \mathrm{T} 158 \mathrm{M}-\mathrm{Tg} / \mathrm{T} 158 \mathrm{M}-$ $\mathrm{Tg}$ animals, respectively. Mice were housed under a 12-hour light/12hour dark cycle, with ad libitum access to food and water.

\section{Nuclear extracts}

Brain nuclear extracts were prepared by dounce homogenization in lysis buffer containing $10 \mathrm{mM}$ HEPES (pH 7.9), 0.5\% NP-40, $1.5 \mathrm{mM} \mathrm{MgCl}_{2}$, and $10 \mathrm{mM} \mathrm{KCl}$. Nuclei were collected, washed, and resuspended in buffer composed of $20 \mathrm{mM}$ HEPES ( $\mathrm{pH} 7.9$ ), $500 \mathrm{mM} \mathrm{KCl,} 1.5 \mathrm{mM} \mathrm{MgCl}_{2}$, $0.2 \mathrm{mM}$ EDTA, and $10 \%$ glycerol. Samples were rotated for 2 hours and ultracentrifuged at $106,000 \mathrm{~g}$ for 30 minutes at $4^{\circ} \mathrm{C}$, and supernatants were subjected to quantitative infrared (IR) Western blotting using the Odyssey Infrared Imaging System (LI-COR Biosciences).

The following antibodies were used in this study: serum directed against the C-terminal region of MeCP2 (55); MeCP2 T158 sitespecific serum (27); $\beta$-actin (Abcam; Ab8226); NeuN (EMD Millipore; 
MAB377); TATA-binding protein (TBP) (Cell Signaling Technology; 8515S); H3 (EMD Millipore; 06-755); GAPDH (Thermo Fisher Scientific; MA5-15738); FLAG (Sigma-Aldrich; F3165); SIN3A (Thermo Fisher Scientific; PA1-870); HDAC3 (Santa Cruz Biotechnology Inc.; SC-11417); and TBLR1 (Bethyl Laboratories; A300-408A).

\section{Subnuclear fractionation}

To prepare nucleoplasm-enriched proteins, cortices were dounce homogenized in $5 \mathrm{ml}$ NE10 buffer (20 mM HEPES, pH 7.5, $10 \mathrm{mM}$ $\mathrm{KCl}, 1 \mathrm{mM} \mathrm{MgCl}, 0.1 \%$ Triton X-100, and $15 \mathrm{mM} \beta$-mercaptoethanol) 30 times using a loose pestle. The resulting nuclei were washed with NE10 buffer and rotated in NE300 buffer (20 mM HEPES, pH 7.5, $300 \mathrm{mM} \mathrm{NaCl}, 10 \mathrm{mM} \mathrm{KCl}, 1 \mathrm{mM} \mathrm{MgCl}, 0.1 \%$ Triton X-100, and $15 \mathrm{mM} \beta$-mercaptoethanol) for 1 hour at $4^{\circ} \mathrm{C}$. Samples were centrifuged at $500 \mathrm{~g}$ for 5 minutes, and the supernatant, which represents the nucleosolic fraction, was collected and saved. The insoluble pellet, consisting of the chromatin-bound fraction, was washed in NE150 buffer and incubated with 500 units of benzonase (Sigma-Aldrich) for 5 minutes at room temperature. The pellet was then resuspended in 50 $\mu$ l NE150 buffer (20 mM HEPES, pH 7.5, $150 \mathrm{mM} \mathrm{NaCl}, 10 \mathrm{mM} \mathrm{KCl}$, $1 \mathrm{mM} \mathrm{MgCl}, 0.1 \%$ Triton X-100, and $15 \mathrm{mM} \beta$-mercaptoethanol) and rotated for 1 hour at $4^{\circ} \mathrm{C}$. Samples were centrifuged at $16,000 \mathrm{~g}$, and the supernatant was collected as the chromatin-bound fraction.

\section{$\mathrm{IHC}$}

IHC was performed essentially as previously described (27). Briefly, mice were deeply anesthetized with $1.25 \%$ (wt/vol) avertin and transcardially perfused with $4 \%$ paraformaldehyde (wt/vol), and tissue was postfixed overnight at $4^{\circ} \mathrm{C}$. Tissue was sectioned coronally or sagittally at a thickness of $20 \mu \mathrm{M}$. IHC was performed using a rabbit anti-serum against MeCP2 (1:1,000) or a rabbit antibody against the FLAG epitope (Sigma-Aldrich; F4725; 1:1,000). All images were acquired using a Leica confocal microscope, with identical settings for laser power, detector gain amplifier offset, and pinhole diameter in each channel.

\section{ChIP}

Mouse cortices were homogenized in buffer containing $100 \mathrm{mM}$ HEPES (pH 7.5), $100 \mathrm{mM} \mathrm{NaCl}, 1 \mathrm{mM}$ EDTA, and 1 mM EGTA and crosslinked for 10 minutes at room temperature with $1 \%$ formaldehyde, followed by quenching with $0.125 \mathrm{M}$ glycine. Cells were lysed in cell lysis buffer (50 mM HEPES, pH 7.5, 140 mM NaCl, 1 mM EDTA, $1 \mathrm{mM}$ EGTA, $0.25 \%$ Triton X-100, 0.5\% NP-40, and 10\% glycerol) for 10 minutes on ice, and nuclei were collected and lysed in $10 \mathrm{mM}$ Tris (pH 8.0), 1\% SDS, 1 mM EDTA, and 1 mM EGTA. The SDS concentration was diluted to $0.625 \%$, and DNA shearing was performed on a Bioruptor instrument (Diagenode; five 10-minute cycles: $30 \mathrm{sec}-$ onds on, 30 seconds off). Buffer conditions were adjusted to contain $150 \mathrm{mM} \mathrm{NaCl}, 0.1 \%$ SDS, and 0.5\% Triton X-100, and chromatin was precleared with Protein A Dynabeads (Invitrogen, Thermo Fisher Scientific). Immunoprecipitation was performed using $32 \mu$ l Protein A Dynabeads and $8 \mu \mathrm{g}$ goat affinity-purified antibody against the C-terminus of MeCP2 or preimmune goat IgG. Beads were washed twice with low-salt buffer (150 mM NaCl, 50 mM HEPES, pH 7.5, 0.1\% sodium deoxycholate [DOC], 1\% Triton X-100, and $1 \mathrm{mM}$ EDTA); once with high-salt buffer (500 mM NaCl, 0.1\% DOC, $50 \mathrm{mM}$ HEPES, $\mathrm{pH} 7.5,1 \%$ Triton X-100, and 1 mM EDTA); once with LiCl buffer (10
mM Tris-HCl, pH 8.1, $250 \mathrm{mM} \mathrm{LiCl,} \mathrm{0.5 \%} \mathrm{NP-40,} \mathrm{0.5 \%} \mathrm{DOC,} \mathrm{and}$ $1 \mathrm{mM}$ EDTA); and twice with Tris-EDTA (TE). Chromatin was eluted with elution buffer (50 mM Tris- $\mathrm{HCl}, \mathrm{pH}$ 8.0, $10 \mathrm{mM}$ EDTA, and $1 \% \mathrm{SDS}$, wt/vol) and reverse cross-linked overnight at $65^{\circ} \mathrm{C}$, followed by treatment with RNase A for 30 minutes at $42^{\circ} \mathrm{C}$ and proteinase $\mathrm{K}$ for 3 hours at $55^{\circ} \mathrm{C}$. DNA was extracted twice with phenol/chloroform and once with chloroform, and ethanol was precipitated. qChIP was performed in duplicate on an ABI 7900 instrument (Thermo Fisher Scientific) using SYBR Green detection. Serial dilutions of input DNA were used to generate a standard curve for each primer pair.

\section{ChIP primers}

The following ChIP primers were used: IAP: forward, $5^{\prime}$-GCTTTCGTTTTTGGGGCTTGG-3'; reverse, 5'-CTTACTCCGCGTTCTCACGAC-3'; LINE-1: forward, 5'-CAATCGCGTGGAACTTGAGAC-3'; reverse, $5^{\prime}$-GACTCAGCTGGCAAGGTAGC-3'; Sst: forward, 5'-CATTGACAGGTACCCAACTGA-3; reverse, 5'-CAGCCACATAGGAGCACACTT-3'; Crh: forward, 5'-TCATAAGAAACCCTTCCATTTTCG-3'; reverse, $5^{\prime}$-TGAATCTCACATCCAATTATATCAACA-3'; $B d n f-6.6 \mathrm{~kb}$ : forward, 5'-CAGCACAGCCTGAAACGTAA-3'; reverse, 5'-TTTGGAGTGGAGCCCTTAGA-3'; Nr4a1 +7.4 kb: forward, 5'-ACTTGGTACCCCCTCCACCTA-3'; reverse, 5'-GCAGGGGTCAGAAAGACAATG-3'; and Gapdh: forward, 5' -TCCCCTCCCCCTATCAGTTC-3; reverse, $5^{\prime}$-GACCCGCCTCATTTTTGAAA-3'.

\section{RT-qPCR}

RNA was extracted with TRIzol Reagent (Invitrogen, Thermo Fisher Scientific) and treated with TURBO DNase (Ambion, Thermo Fisher Scientific). Reverse transcription reactions were performed with Superscript III (Invitrogen, Thermo Fisher Scientific) using random hexamer priming. Results were quantified on an ABI 7900 system using SYBR Green technology. All RNA expression levels were normalized to Gapdh.

\section{RT-qPCR primers}

The following RT-PCR primers were used: Mecp2: forward, $5^{\prime}$-CATACATAGGTCCCCGGTCA-3'; reverse, 5'-CAGGCAAAGCAGAAACATCA-3'; Gapdh: forward, 5'-GATGCCCCCATGTTTGTGAT-3'; reverse, 5'-GGTCATGAGCCCTTCCACAAT-3'; MECP2: forward, 5'-TTCACGGTAACTGGGAGAGG-3'; reverse, 5-GGAGCTTCCCAGGACTTTTC-3'; and GAPDH: forward, 5'-GAAATCCCATCACCATCTTCCAGG-3'; reverse, 5' -GAGCCCCAGCCTTCTCCATG-3'.

\section{Co-IP}

Co-IPs were performed as described previously, with some modifications (7). Briefly, hindbrains were dounce homogenized in $5 \mathrm{ml} \mathrm{NE10}$ buffer (20 mM HEPES, pH 7.5, $10 \mathrm{mM} \mathrm{KCl,} 1 \mathrm{mM} \mathrm{MgCl}$, 0.1\% Triton $\mathrm{X}-100$, and $15 \mathrm{mM} \beta$-mercaptoethanol), 10 times using a loose pestle and 10 times using a tight pestle. Nuclei were pelleted, washed, and incubated with 250 units benzonase (Sigma-Aldrich) for 5 minutes at room temperature. Nuclei were then resuspended in $1 \mathrm{ml} \mathrm{NE150} \mathrm{buf-}$ fer (20 mM HEPES, pH 7.5, $150 \mathrm{mM} \mathrm{NaCl}, 10 \mathrm{mM} \mathrm{KCl,} 1 \mathrm{mM} \mathrm{MgCl}$, $0.1 \%$ Triton $\mathrm{X}-100$, and $15 \mathrm{mM} \beta$-mercaptoethanol) and rotated for 2 hours at $4^{\circ} \mathrm{C}$. Lysates were centrifuged at $16,000 \mathrm{~g}$, and the supernatant was precleared for 2 hours with protein A agarose beads. Precleared supernatants were incubated with Anti-FLAG M2 Affinity Gel (Sigma-Aldrich) overnight. Bead-bound immune complexes were 
washed with buffer composed of $20 \mathrm{mM}$ HEPES (pH 7.5), $150 \mathrm{mM}$ $\mathrm{NaCl}, 0.1 \%$ Triton X-100, 10\% glycerol, and 0.2 mM EDTA. FLAG proteins and their interacting proteins were eluted with $100 \mu \mathrm{g} / \mathrm{ml}$ FLAG peptide for 1 hour at $4^{\circ} \mathrm{C}$ and resolved via SDS-PAGE.

\section{Primary neuronal culture}

Cortices from PO/P1 pups were dissected under a stereoscope and digested with trypsin $(0.25 \% \mathrm{wt} / \mathrm{vol})$ for 20 minutes at $37^{\circ} \mathrm{C}$. The trypsin was inactivated with plating media, and cortices were triturated and plated at a density of $1.0 \times 10^{6}$ to $1.5 \times 10^{6}$ cells per well of a 12 -well dish coated with $0.01 \%$ poly-L-lysine (Sigma-Aldrich). Cultures were maintained in neurobasal medium supplemented with B27, 1× Gluta$\max$ (Gibco, Thermo Fisher Scientific), 0.5\% D-glucose, $100 \mu \mathrm{g} / \mathrm{ml}$ Primocin (InvivoGen), and 10\% FBS.

After 3 days in vitro (DIV), cells were treated with $100 \mu \mathrm{g} / \mathrm{ml}$ cycloheximide or $20 \mu \mathrm{M}$ MG132 for the indicated durations (Supplemental Figure $2 \mathrm{C}$ and Figure 6G) and harvested. Cells were lysed in NE10

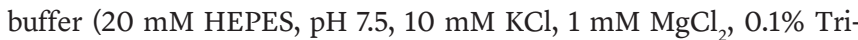
ton $\mathrm{X}-100$, and $15 \mathrm{mM} \beta$-mercaptoethanol). The resulting nuclei were incubated with NE300 buffer (20 mM HEPES, pH 7.5, $300 \mathrm{mM} \mathrm{NaCl}$, $10 \mathrm{mM} \mathrm{KCl}, 1 \mathrm{mM} \mathrm{MgCl}, 0.1 \%$ Triton X-100, and $15 \mathrm{mM} \beta$-mercaptoethanol) for 1 hour on ice. Samples were centrifuged at $500 \mathrm{~g}$ for 5 minutes, the supernatant was collected, and the proteins were resolved via SDS-PAGE.

\section{Cell culture}

Mouse N2A neuroblastoma and HEK 293T cells were cultured in DMEM containing 10\% FBS (Invitrogen, Thermo Fisher Scientific), $2 \%$ penicillin-streptomycin, and $1 \%$ sodium pyruvate.

\section{Constructs}

The MeCP2 LEMPRA (lentivirus-mediated protein-replacement assay) plasmid has been described previously (55). Modifications to the construct included the removal of the GFP expression cassette and incorporation of an N-terminal FLAG tag and a C-terminal Dendra2 fusion in-frame with the MeCP2 coding sequence. QuikChange site-directed mutagenesis was used to introduce the MeCP2 T158M mutation.

\section{Lentivirus production}

Lentiviruses were produced by cotransfection of HEK293T cells with the FLAG-MeCP2-Dendra2 or FLAG-T158M-Dendra2 plasmid, together with the helper plasmids $\Delta 8.9$ and VSV-G. N2a cells were seeded in a 6 -well dish at a concentration of $1 \times 10^{5}$ cells $/ \mathrm{ml}$. The following day, $1 \mathrm{ml}$ filtered virus was added to each well, together with 2 $\mathrm{ml}$ fresh media and $16 \mu \mathrm{g}$ polybrene and incubated at $37^{\circ} \mathrm{C}$. The media were changed 24 hours later, and the next day, lentivirus-infected N2a cells were singly sorted according to GFP into 96-well plates and expanded to generate clonal cell lines.

\section{Immunofluorescence}

N2a lines stably expressing FLAG-MeCP2-Dendra2 or FLAG-T158MDendra2 were grown on gelatin-coated coverslips. Cells were washed in PBS and fixed in $4 \%$ formaldehyde for 20 minutes at room temperature. Fixed cells were then briefly washed (0.1\% BSA in PBS), blocked (1\% BSA and 0.5\% Tween-20) for 1 hour, and incubated with Topro3 (diluted at 1:1,000 in PBS) for 15 minutes. After washing in PBS, coverslips were mounted facedown onto slides.

\section{Plethysmography}

A whole-body plethysmograph (Buxco II; DSI) allowed mice to assume natural postures and movement, during which apneas and respiratory regularity were assessed. The plethysmograph system, calibrated to $1-\mathrm{ml}$ volume, provided constant air exchange at 1 liter/minute. Ambient temperatures within the chamber ranged between $24^{\circ} \mathrm{C}$ and $24.5^{\circ} \mathrm{C}$. Mice were given 20 minutes to explore the chamber and were then recorded for 20 minutes of quiet wakefulness breathing. Airflow signals were recorded and analyzed using FinePointe Software (version 2.0.3.1; DSI). Apneas were defined as a cessation of airflow for more than 1 second (37) and were analyzed as the total number of apneas/time, expressed as apneas/hour. The respiratory frequency irregularity was defined as previously described (56) using the variance of the absolute values of $\mathrm{T}_{\text {TOTn }}-\mathrm{T}_{\mathrm{TOTn}+1} / \mathrm{T}_{\text {TOTn }+1}$, where $\mathrm{T}_{\text {тОт }}$ equals the total respiratory cycle time.

\section{ERP recordings}

Each mouse was deeply anesthetized (1\%-2\% isoflurane) and mounted in a stereotaxic frame with nonpuncturing ear bars. Three stainless steel electrodes, mounted in a single headstage, were aligned to the sagittal axis of the skull. A stainless steel recording electrode was placed $2.0 \mathrm{~mm}$ posteriorly and $2.0 \mathrm{~mm}$ left laterally relative to bregma, at a depth of $1.8 \mathrm{~mm}$. Ground and reference electrodes were placed anterior to the hippocampal electrode at distances of $1.0 \mathrm{~mm}$ and $2.0 \mathrm{~mm}$, respectively. The headstage was then fixed to the skull with screws and dental acrylic.

ERP recordings were performed on freely mobile, nonanesthetized 10- to 14-week-old mice in their home cage environment after a 20-minute acclimation to the recording room. For local field potential recordings, neural signals were acquired using a Cheetah Data Acquisition System (Neuralynx; Digital Lynx 4SX; $2.0 \mathrm{kHz}$ sample rate). Auditory stimuli consisting of a series of 250 whitenoise pips (10-ms duration, $85-\mathrm{dB}$ sound pressure level [SPL], 0.25 $\mathrm{Hz}$ frequency) were presented through speakers on the recording chamber ceiling. Stimuli were calibrated using a sound pressure meter. Because of the frequency response of speakers, white noise has a corresponding bandwidth of 700 to $20,000 \mathrm{kHz}$. Analyses of event-related power and PLF were performed in a manner similar to that described previously using wavelet methods with custom C routines (41).

For statistical analysis, event-related power and PLF were separated into frequency ranges, with the mean power or PLF calculated between frequencies: $\delta, 2-4 \mathrm{~Hz} ; \theta, 4-8 \mathrm{~Hz} ; \alpha, 8-12 \mathrm{~Hz} ; \beta, 12-30 \mathrm{~Hz}$;

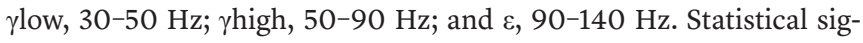
nificance was assessed using permutation tests based on $t$ statistics and false-discovery correction made using the $q$-value methodology as previously described (57).

\section{Phenotypic scoring}

Mice were monitored for the development of RTT-like phenotypes as described previously (15).

\section{Animal behavior}

All animal behavioral studies were performed by female handlers blinded to genotype. Mice were allowed to habituate to the testing room for at least 30 minutes prior to testing, with each test performed at the same time of day. 
Open field assay. Locomotor activity was measured by beam breaks in an arena with a $16 \times 16$ photobeam array (San Diego Instruments). Mice were placed in the middle of the arena, and the number of beam breaks, distance travelled, and average speed were quantified over the course of 10 minutes in 1-minute bins. Testing was performed under dim lighting conditions (15 lux).

Accelerating rotarod. Mice were placed on a rotarod apparatus (Harvard Apparatus), accelerating linearly from 4 to $40 \mathrm{rpm}$ for 5 trials a day on 4 consecutive days. Each mouse was allowed at least 10 minutes of rest between each trial, with each trial lasting a maximum of 5 minutes. The amount of time each mouse spent on the rod before falling was recorded for each trial.

Treadmill gait analysis. Gait behaviors were assayed using the TreadScan System (Clever Sys Inc.). Prior to testing, animals were habituated to the testing room for at least 30 minutes and were allowed to habituate in the treadmill chamber for 3 minutes. The mice were tested for 20 seconds at a constant speed of $18 \mathrm{~cm} / \mathrm{s}$. Animals who could not maintain this walking speed for the entire 20 -second duration were excluded from the study. The accompanying TreadScan software automatically analyzed gait characteristics.

\section{Statistics}

Data are presented as the mean \pm SEM. Statistical analyses were performed using GraphPad Prism 6 (GraphPad Software). Brain weight comparisons between WT and T158M-knockin mice were analyzed using a 2-tailed $t$ test with Bonferroni's correction. Body weight and phenotypic scores for Mecp $2^{T 158 M}$ mice versus WT controls were analyzed using a 2-tailed $t$ test. MeCP2 protein levels with developmental age were analyzed using a 2-tailed $t$ test. The following measurements were analyzed using 1-way ANOVA $(P<0.05)$, followed, when appropriate, by Tukey's post-hoc test: ChIP-qPCR; total MeCP2 levels, body weight, and brain weight for Mecp $2^{+/ y}, M e c p 2^{+/ y} \mathrm{~T} 158 \mathrm{M}-\mathrm{Tg}, M e c p 2^{\mathrm{T} 158 \mathrm{M} / \mathrm{y}}$, and Mecp $2^{\text {T158M/y }} \mathrm{T} 158 \mathrm{M}$-Tg animals; number of beam breaks, distanced traveled, and average speed in the open field assay; stance and propulsion times; and breathing irregularities and number of apneas per hour. The rotarod task was analyzed using 2-way ANOVA $(P<0.05)$. Comparison of MeCP2 levels in nucleosolic versus chromatin fractions was performed using 2-way ANOVA, followed by Sidak's post-hoc test. MeCP2 protein levels following MG132 treatment were analyzed using 1-way ANOVA, followed by Dunnett's multiple comparisons test.

\section{Study approval}

Experiments were conducted in accordance with the ethical guidelines of the NIH and with the approval of the IACUC of the University of Pennsylvania.

\section{Acknowledgments}

We thank the Intellectual and Developmental Disability Research Center Gene Manipulation Core at the Children's Hospital of Boston (M. Thompson) for the generation of knockin mice and the Transgenic and Chimeric Core Facility at the University of Pennsylvania (J. Richa) for the generation of Tg mice. This work was supported by grants from the NIH (R01 NS081054 and R01 MH091850, to ZZ) and a Basic Research Award (to ZZ) and a Mentored Training Fellowship (to JML), both from RettSyndrome. org. ZZ is a Pew Scholar in Biomedical Sciences.

Address correspondence to: Zhaolan Zhou, Department of Genetics, University of Pennsylvania Perelman School of Medicine, 415 Curie Boulevard, Philadelphia, Pennsylvania 19104, USA. Phone: 215.746.5025; E-mail: zhaolan@mail.med.upenn.edu.

DG's present address is: Department of Biology, University of York, Heslington, York, United Kingdom.
1. Chahrour M, Zoghbi HY. The story of Rett syndrome: from clinic to neurobiology. Neuron. 2007;56(3):422-437

2. Amir RE, Van den Veyver IB, Wan M, Tran CQ, Francke U, Zoghbi HY. Rett syndrome is caused by mutations in X-linked MECP2, encoding methyl-CpG-binding protein 2. Nat Genet. 1999;23(2):185-188.

3. Lewis JD, et al. Purification, sequence, and cellular localization of a novel chromosomal protein that binds to methylated DNA. Cell. 1992;69(6):905-914.

4. Nan X, Campoy FJ, Bird A. MeCP2 is a transcriptional repressor with abundant binding sites in genomic chromatin. Cell. 1997;88(4):471-481.

5. Jones PL, et al. Methylated DNA and MeCP2 recruit histone deacetylase to repress transcription. Nat Genet. 1998;19(2):187-191.

6. Stancheva I, Collins AL, Van den Veyver IB, Zoghbi $\mathrm{H}$, Meehan RR. A mutant form of MeCP2 protein associated with human Rett syndrome cannot be displaced from methylated DNA by notch in Xenopus embryos. Mol Cell. 2003;12(2):425-435.

7. Lyst MJ, et al. Rett syndrome mutations abolish the interaction of MeCP2 with the NCoR/SMRT co-repressor. Nat Neurosci. 2013;16(7):898-902.

8. Chahrour M, et al. MeCP2, a key contributor to neurological disease, activates and represses tran- scription. Science. 2008;320(5880):1224-1229.

9. Ben-Shachar S, Chahrour M, Thaller C, Shaw $\mathrm{CA}$, Zoghbi HY. Mouse models of MeCP2 disorders share gene expression changes in the cerebellum and hypothalamus. Hum Mol Genet. 2009;18(13):2431-2442.

10. Zhao YT, Goffin D, Johnson BS, Zhou Z. Loss of $\mathrm{MeCP} 2$ function is associated with distinct gene expression changes in the striatum. Neurobiol Dis. 2013;59:257-266.

11. Sugino K, et al. Cell-type-specific repression by methyl-CpG-binding protein 2 is biased toward long genes. J Neurosci. 2014;34(38):12877-12883.

12. Guo JU, et al. Distribution, recognition and regulation of non-CpG methylation in the adult mammalian brain. Nat Neurosci. 2014;17(2):215-222.

13. Gabel HW, et al. Disruption of DNA-methylation-dependent long gene repression in Rett syndrome. Nature. 2015;522(7554):89-93.

14. Chen L, et al. MeCP2 binds to non-CG methylated DNA as neurons mature, influencing transcription and the timing of onset for Rett syndrome. Proc Natl Acad Sci U S A. 2015;112(17):5509-5514.

15. Guy J, Gan J, Selfridge J, Cobb S, Bird A. Reversal of neurological defects in a mouse model of Rett syndrome. Science. 2007;315(5815):1143-1147.

16. Garg SK, et al. Systemic delivery of MeCP2 res- cues behavioral and cellular deficits in female mouse models of Rett syndrome. J Neurosci. 2013;33(34):13612-13620.

17. Van Esch H, et al. Duplication of the MECP2 region is a frequent cause of severe mental retardation and progressive neurological symptoms in males. Am J Hum Genet. 2005;77(3):442-453.

18. Meins M, et al. Submicroscopic duplication in Xq28 causes increased expression of the MECP2 gene in a boy with severe mental retardation and features of Rett syndrome. JMed Genet. 2005;42(2):e12.

19. Collins AL, et al. Mild overexpression of MeCP2 causes a progressive neurological disorder in mice. Hum Mol Genet. 2004;13(21):2679-2689.

20. Samaco RC, et al. A partial loss of function allele of methyl-CpG-binding protein 2 predicts a human neurodevelopmental syndrome. Hum Mol Genet. 2008;17(12):1718-1727.

21. Kerr B, Alvarez-Saavedra M, Sáez MA, Saona A, Young JI. Defective body-weight regulation, motor control and abnormal social interactions in Mecp2 hypomorphic mice. Hum Mol Genet. 2008;17(12):1707-1717.

22. Lombardi LM, Baker SA, Zoghbi HY. MECP2 disorders: from the clinic to mice and back. J Clin Invest. 2015;125(8):2914-2923.

23. Neul JL, et al. Specific mutations in methylCpG-binding protein 2 confer different 
severity in Rett syndrome. Neurology. 2008;70(16):1313-1321.

24. Chen RZ, Akbarian S, Tudor M, Jaenisch R. Deficiency of methyl-CpG binding protein- 2 in CNS neurons results in a Rett-like phenotype in mice. Nat Genet. 2001;27(3):327-331.

25. Guy J, Hendrich B, Holmes M, Martin JE, Bird A. A mouse Mecp2-null mutation causes neurological symptoms that mimic Rett syndrome. Nat Genet. 2001;27(3):322-326.

26. Ho KL, McNae IW, Schmiedeberg L, Klose RJ, Bird AP, Walkinshaw MD. MeCP2 binding to DNA depends upon hydration at methyl-CpG. Mol Cell. 2008;29(4):525-531.

27. Goffin D, et al. Rett syndrome mutation MeCP2 T158A disrupts DNA binding, protein stability and ERP responses. Nat Neurosci. 2011;15(2):274-283.

28. Brown K, et al. The molecular basis of variable phenotypic severity among common missense mutations causing Rett syndrome. $\mathrm{Hum} \mathrm{Mol}$ Genet. 2016;25(3):558-570.

29. Skene PJ, et al. Neuronal MeCP2 is expressed at near histone-octamer levels and globally alters the chromatin state. Mol Cell. 2010;37(4):457-468.

30. Cohen S, et al. Genome-wide activity-dependent MeCP2 phosphorylation regulates nervous system development and function. Neuron. 2011;72(1):72-85.

31. Ghosh RP, Horowitz-Scherer RA, Nikitina T, Gierasch LM, Woodcock CL. Rett syndrome-causing mutations in human $\mathrm{MeCP} 2$ result in diverse structural changes that impact folding and DNA interactions. J Biol Chem. 2008;283(29):20523-20534.

32. Borchelt DR, et al. A vector for expressing foreign genes in the brains and hearts of transgenic mice. Genet Anal. 1996;13(6):159-163.

33. Kriaucionis S, Bird A. The major form of MeCP2 has a novel $\mathrm{N}$-terminus generated by alternative splicing. Nucleic Acids Res. 2004;32(5):1818-1823.

34. Mnatzakanian GN, et al. A previously unidentified MECP2 open reading frame defines a new protein isoform relevant to Rett syndrome. Nat Genet. 2004;36(4):339-341.
35. Yasui DH, et al. Mice with an isoform-ablating Mecp2 exon 1 mutation recapitulate the neurologic deficits of Rett syndrome. Hum Mol Genet. 2014;23(9):2447-2458.

36. Derecki NC, et al. Wild-type microglia arrest pathology in a mouse model of Rett syndrome. Nature. 2012;484(7392):105-109.

37. Bissonnette JM, Schaevitz LR, Knopp SJ, Zhou Z. Respiratory phenotypes are distinctly affected in mice with common Rett syndrome mutations MeCP2 T158A and R168X. Neuroscience. 2014;267:166-176.

38. Bader GG, Witt-Engerström I, Hagberg B. Neurophysiological findings in the Rett syndrome, II: Visual and auditory brainstem, middle and late evoked responses. Brain Dev. 1989;11(2):110-114.

39. Stauder JE, Smeets EE, van Mil SG, Curfs LG. The development of visual- and auditory processing in Rett syndrome: an ERP study. Brain Dev. 2006;28(8):487-494.

40. Durand S, et al. NMDA receptor regulation prevents regression of visual cortical function in the absence of Mecp2. Neuron. 2012;76(6):1078-1090.

41. Goffin D, Brodkin ES, Blendy JA, Siegel SJ, Zhou Z. Cellular origins of auditory event-related potential deficits in Rett syndrome. Nat Neurosci. 2014;17(6):804-806.

42. Katz DM, et al. Preclinical research in Rett syndrome: setting the foundation for translational success. Dis Model Mech. 2012;5(6):733-745.

43. Buchovecky CM, et al. A suppressor screen in Mecp2 mutant mice implicates cholesterol metabolism in Rett syndrome. Nat Genet. 2013;45(9):1013-1020.

44. Gadalla KK, Ross PD, Riddell JS, Bailey ME, Cobb SR. Gait analysis in a Mecp2 knockout mouse model of Rett syndrome reveals earlyonset and progressive motor deficits. PLOS ONE. 2014;9(11):e112889.

45. Chen WG, et al. Derepression of BDNF transcription involves calcium-dependent phosphorylation of MeCP2. Science. 2003;302(5646):885-889.

46. Castro J, et al. Functional recovery with recombinant human IGF1 treatment in a mouse model of Rett Syndrome. Proc Natl Acad Sci U S A. 2014;111(27):9941-9946.

47. Popescu AC, Sidorova E, Zhang G, Eubanks JH. Aminoglycoside-mediated partial suppression of MECP2 nonsense mutations responsible for Rett syndrome in vitro. J Neurosci Res. 2010;88(11):2316-2324.

48. Pitcher MR, et al. Rett syndrome like phenotypes in the R255X Mecp2 mutant mouse are rescued by MECP 2 transgene. Hum Mol Genet. 2015;24(9):2662-2672.

49. Ballas N, Lioy DT, Grunseich C, Mandel G. Noncell autonomous influence of MeCP2-deficient glia on neuronal dendritic morphology. Nat Neurosci. 2009;12(3):311-317.

50. Maezawa I, Swanberg S, Harvey D, LaSalle JM, Jin LW. Rett syndrome astrocytes are abnormal and spread MeCP2 deficiency through gap junctions. J Neurosci. 2009;29(16):5051-5061.

51. Abdala AP, Dutschmann M, Bissonnette JM, Paton JF. Correction of respiratory disorders in a mouse model of Rett syndrome. Proc Natl Acad Sci U S A. 2010;107(42):18208-18213.

52 . Weese-Mayer DE, et al. Autonomic nervous system dysregulation: breathing and heart rate perturbation during wakefulness in young girls with Rett syndrome. Pediatr Res. 2006;60(4):443-449.

53. Wegener E, Brendel C, Fischer A, Hülsmann S, Gärtner J, Huppke P. Characterization of the MeCP2R168X knockin mouse model for Rett syndrome. PLoS One. 2014;9(12):e115444.

54. Julu PO, et al. Characterisation of breathing and associated central autonomic dysfunction in the Rett disorder. Arch Dis Child. 2001;85(1):29-37.

55. Zhou Z, et al. Brain-specific phosphorylation of MeCP2 regulates activity-dependent Bdnf transcription, dendritic growth, and spine maturation. Neuron. 2006;52(2):255-269.

56. Viemari JC, et al. Mecp2 deficiency disrupts norepinephrine and respiratory systems in mice. J Neurosci. 2005;25(50):11521-11530.

57. Storey JD, Tibshirani R. Statistical significance for genomewide studies. Proc Natl Acad Sci U S A. 2003;100(16):9440-9445. 